\title{
Systèmes ludo-persuasifs pour la consommation durable. 1 - Points de repères et défis à relever
}

\begin{abstract}
Bernard SENACH
INRIA Sophia Antipolis

Méditerrannée

bernard.senach@inria.fr

Anne-Laure NEGRI

Ethnomining

alnegri@ethnomining.com

\author{
Ludo-persuasive systems for sustainable consumption. \\ 1 - Landmarks and challenges
}

Abstract. For over 40 years, it is common knowledge that industrial society has to reduce its energy consumption. Most of people are now aware that this change is necessary. However, commitment to action is still difficult and there is substantial work to be done. Attention has turned to Human and Social Sciences, as a deeper understanding of behaviors' determinant and use of influence principles could help to speed up behavior changes. New technical devices combining game design, interaction techniques and persuasion have emerged but the field is still in its infancy. This article gives a glimpse at a toolbox helping to design and evaluate interactive persuasive devices and discuss five main challenges. This work is extended by two others papers: Negri et Senach (2015b) provide a first grid of persuasion principles and in Senach et Negri (2015c), these grid have been applied to assess the persuasive properties of an energy challenge within a company.

Key words: energy saving, sustainable development, gamification, behavior changes.

Résumé. Depuis plus de 40 ans, il est de notoriété publique qu'il est nécessaire de modifier les comportements de consommation énergétique dans les sociétés industrielles. Globalement, la prise de conscience par le grand public de la gravité des questions d'écologie est maintenant acquise. Et pourtant, le passage à l'acte est encore difficile et les gisements d'économies restent considérables. La lenteur des évolutions comportementales a conduit à rechercher du côté des Sciences Humaines et Sociales des modèles permettant une compréhension en profondeur des déterminants des comportements et à identifier des principes d'influence (crédibilité, expertise, réciprocité, etc.). L'implémentation de ces principes dans des dispositifs interactifs est une solution qui pourrait accompagner les changements de comportements ; ces nouveaux systèmes, associant le plaisir du jeu et les techniques de persuasion, baptisés ici "systèmes ludo-persuasifs " (SLP) pourraient être de bons candidats pour aider à l'adoption d'éco-gestes. Mais, l'utilisation des technologies interactives pour la persuasion est encore trop récente pour qu'une véritable ingénierie se soit développée. Dans le travail présenté ci-dessous, nous proposons l'élaboration d'une « boîte à outils » ludo-persuasive en discutant les défis qui doivent être relevés. Cet article est prolongé par deux travaux complémentaires : Negri et Senach (2015b) proposent une structuration de principes persuasifs et ils appliquent ensuite cette grille pour analyser a posteriori les caractéristiques ludo-persuasives d'un challenge énergétique conduit en entreprise (Senach \& Negri, 2015c).

Mots-clés : économies d'énergie, développement durable, ludification, changement comportemental.
\end{abstract}




\section{DES TEChNOLOGIES PERSUASIVES AUX SYSTEMES LUDO-PERSUASIFS}

\subsection{Pérennité du besoin de changement}

L'intérêt pour les techniques d'influence et de persuasion n'est pas nouveau : Aristote a fondé l'art de la persuasion sur la crédibilité de l'orateur (ethos), la logique de son discours (logos) et sa capacité à susciter l'émotion chez l'auditeur (pathos). Depuis, les connaissances dans ce domaine se sont sans cesse affinées. À l'époque moderne, un nombre considérable de travaux ont été conduits dans les Sciences Humaines et Sociales (SHS) pour identifier les déterminants du changement d'attitudes et de comportements. Audelà des objectifs de manipulation des foules par la propagande ou des préoccupations commerciales de développement des ventes, la volonté d'orienter les comportements des individus est maintenant liée à la nécessité de les faire évoluer vers une consommation soutenable.

Des efforts considérables ont été entrepris pour effectuer une "conversion de masse » à la consommation durable, par exemple en exhortant les citoyens aux économies d'énergie, en les incitant au tri des déchets, à l'usage d'une mobilité douce, et de nombreux dispositifs ont été élaborés pour les aider. Les premières campagnes d'information ciblées remontent au premier choc pétrolier, et depuis 1971, elles ont fait preuve de toujours plus d'inventivité ${ }^{1}$. Globalement, la prise de conscience par le grand public de la gravité des questions d'écologie est acquise et par exemple, en 2002, il est ressorti d'une enquête du Credoc sur les "Conditions de vie et les Aspirations des français ", que "l'écologie est, sans l'ombre d'un doute, une préoccupation de l'ensemble de la population : neuf personnes sur dix se disent "assez " ou " très " sensibles aux questions environnementales " ${ }^{2}$. Dix ans plus tard, en 2012, 93\% des citoyens pensent qu'ils peuvent avoir un impact important sur l'environnement. Et pourtant, le passage à l'acte est encore difficile et les gisements d'économies restent considérables. Une des interprétations les plus fréquemment avancées pour expliquer ces difficultés concerne la « résistance au changement " des individus, phénomène psychologique qui les pousserait à maintenir un statu quo en préférant le conservatisme et l'immobilisme. Pour aider à dépasser les blocages, les travaux théoriques consacrés aux problématiques de l'influence et de l'engagement ont été renouvelés et enrichis par des approches parfois plus pragmatiques telles que celle de l'économie comportementale (Thaler \& Sunstein, 2008) qui met en œuvre des "coups de pouce » (nudges) orientant les individus vers le changement désiré. Les avancées réalisées dans le domaine des Sciences et Technologies de l'Information et de la Communication (STIC) ont aussi ouvert la nouvelle perspective des technologies persuasives.

\subsection{Technologies persuasives}

L'union des technologies informatiques développées dans les STIC et des principes de persuasion identifiées par les SHS, célébrée en 2003 par $\mathrm{Fogg}^{3}$ a donné naissance au nouveau champ des recherches des technologies persuasives. Dénommée pendant un temps " captologie », cette union, qui pourrait évoquer celle de la carpe et du lapin, permet

\footnotetext{
1 Voir par exemple : http://www.youtube.com/watch?feature=player_embedded\&v=Wq40n9BtpRk

et http://www.sircome.fr/Stop-aux-trajets-ridicules-en

2 http://www.credoc.fr/pdf/Sou/opinions_environnement.pdf

${ }^{3}$ Fogg a proposé initialement une liste de 46 principes implémentables distribués en 8 classes selon qu'ils concernent, entre autres, la simplification des tâches, la crédibilité du système, le rôle social de l'ordinateur ou la dynamique temporelle de la persuasion (Kairos).
} 
d'enrichir l'arsenal des ressources d'influence et de persuasion des sciences humaines avec des techniques de contrôle du comportement des utilisateurs de systèmes interactifs très élaborées.

Comme l'a souligné Fogg (2003), au-delà de sa capacité de calcul et ses algorithmes qui permettent, par exemple, de personnaliser une recommandation, un ordinateur est également un dispositif fiable, persévérant, pouvant jouer un rôle de confident ou de coach, susceptible de recueillir des données intimes et d'interagir via de nombreuses "modalités d'expression » (images, messages vocaux, email, SMS...). Ainsi, l'utilisateur d'un dispositif de persuasion technologique est en mesure d'entretenir avec celui-ci une relation durable, quasi interpersonnelle. De plus, les technologies permettant désormais le suivi des comportements en temps réel et en mobilité, l'exploitation de données contextualisées (context awareness) permet d'assurer une continuité de services.

On parle donc de technologie persuasive pour qualifier tout système technique conçu intentionnellement pour influencer avec succès le comportement ou les attitudes de ses utilisateurs (Kaptein et al., 2010). Cette volonté explicite d'orienter le comportement des usagers voire de le modifier de façon plus durable pose naturellement beaucoup de questions éthiques ${ }^{4}$ liées notamment au développement du commerce en ligne, mais les techniques de persuasion peuvent aussi être utilisées pour la "bonne cause " et avoir un rôle à jouer pour cadrer la consommation énergétique ou alimentaire, l'organisation du travail, des transports, voire celle des villes. Le recours aux technologies persuasives pour changer les modes de vie constitue ainsi une piste qui, de fait, est explorée depuis quelques années Pour un aperçu du développement des applicatifs de persuasion, le lecteur est renvoyé à Bastien (2012). Une revue des articles présentés lors des conférences Persuasive Design permet aussi d'appréhender l'état de l'art dans cette discipline.

Outre l'implémentation de petits coups de pouce, de trucs et astuces opportunistes d'incitation au changement, les systèmes persuasifs bénéficient maintenant de la convergence récente des technologies persuasives avec celles du jeu. On parle alors de dispositifs « ludo-persuasifs».

\section{3 Évolution vers des systèmes ludo-persuasifs}

On appelle ici système ludo-persuasif (SLP) tout système interactif dont l'objectif est d'inciter ses utilisateurs à adopter un comportement déterminé et qui, pour atteindre cet objectif, s'appuie sur des principes de persuasion et des mécanismes de jeu. Dans l'idéal, au travers d'interactions sans frictions, un système ludo-persuasif vise à modifier une attitude et/ou un comportement, sans coercition, et ce, de manière plaisante. Ce rapprochement est très discuté dans la littérature car l'application des principes de jeu dans les applications professionnelles reste souvent très superficielle, sans que l'on se préoccupe outre mesure de l'expérience utilisateur qui en résulte ${ }^{5}$. Cette utilisation sans discernement de recettes de " pointification ${ }^{6}$ " et de " badgification ${ }^{7}$ " revient pour Kumar et Herger (2013) à " napper un brocoli avec du chocolat ". Bien que la discipline soit récente, beaucoup de travaux ont déjà été engagés, mais le domaine est trop jeune pour apporter des réponses claires et définitives aux questions concernant le rôle que ces systèmes peuvent jouer dans la consommation durable. Les quelques évaluations effectuées (Hamari et al., 2014) laissent penser que les marges de progression sont encore importantes. Sans vouloir établir un état de l'art approfondi, on donne ci-dessous un rapide aperçu de la structuration des champs de

\footnotetext{
${ }^{4}$ Voir par exemple à ce sujet Atkinson, (2006) et Némery (2012) qui cite notamment Berdichevsky et Neuenschwander (1999)

5 Voir par exemple http://www.hideandseek.net/2010/10/06/cant-play-wont-play/

6 Incrémentation du nombre de points gagnés par le joueur en cas de réussite.

${ }^{7}$ Attribution au joueur d'une récompense symbolique (badge) représentant le niveau de performance atteint.
} 
recherche concernés. La maturité du champ passera par le développement d'une ingénierie des systèmes ludo-persuasifs mais la route est longue et pleine d'embuches. Les sections cidessous veulent apporter une contribution modeste à la feuille de route en proposant tout d'abord des points de repère pour comprendre l'organisation du champ de recherche avant de discuter quelques-uns des défis à relever.

\section{POINTS DE REPERES POUR ABORDER LES SYSTEMES LUDO PERSUASIFS}

La conception de dispositifs ludo-persuasifs se situe dans le cadre global de la " conception intentionnelle » (Design with Intent), bien illustrée par les travaux de Lockton et al. (2010). La démarche adoptée consiste à mettre en œuvre lors de la conception des "'incitateurs"8 destinés à orienter les comportements, en s'appuyant plus ou moins directement sur des modèles d'influence dérivés des SHS. On peut en trouver des exemples dans les aménagements de l'espace destinés à organiser des flux de déplacements, (voir par exemple The fun theory http://www.thefuntheory.com/), ou bien en architecture, dans le design de produits et également dans la conception d'interfaces utilisateurs. Dans ce dernier contexte, on parle parfois d'ergonomie incitative (Némery, 2012) pour caractériser les aménagements spécifiques réalisés dans les sites de e-commerce pour orienter les choix et faciliter les conversions (boutons "call to action", présentation d'options d'achats, etc.).

\subsection{Convergence de travaux}

Trois champs de recherche contribuent au développement des technologies ludopersuasives : celui des jeux, celui de la persuasion en Sciences humaines et Sociales (SHS), et celui des technologies interactives en Sciences et Technologies de l'Information et de la Communication (STIC). Ils sont représentés sur le schéma ci-dessous.

\section{Figure 1: Champs de recherche contribuant au développement des systèmes ludo-persuasifs}

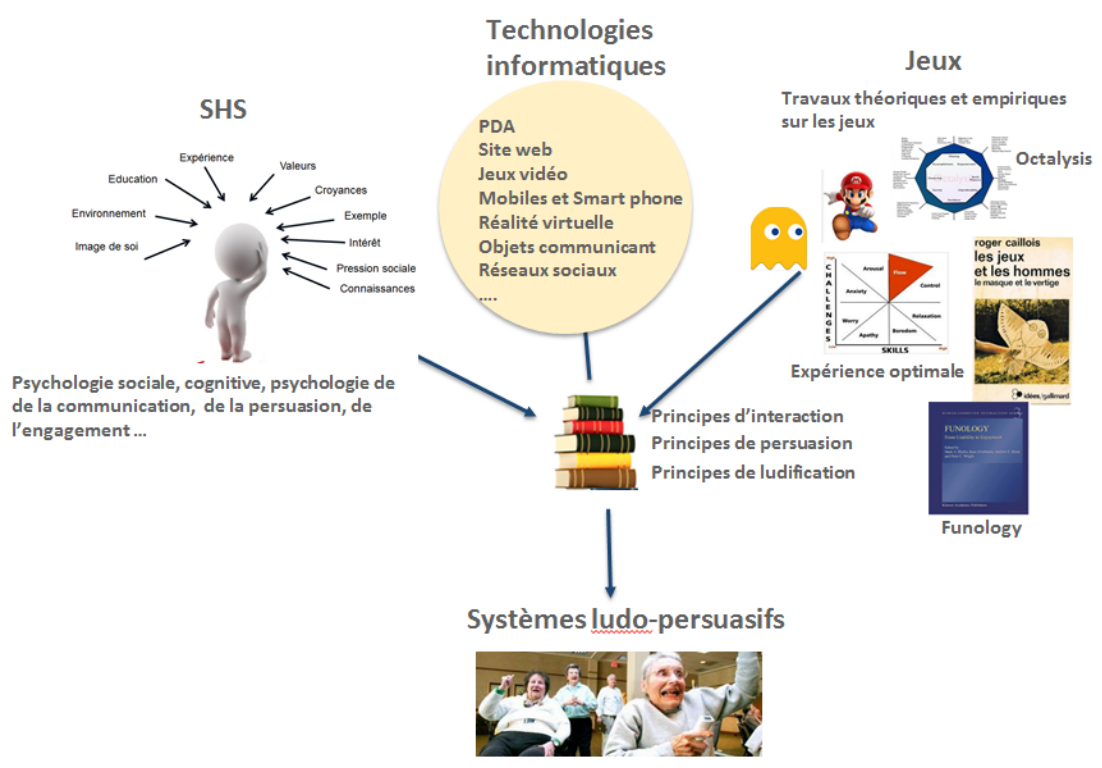

Le développement des jeux vidéo a dynamisé les travaux consacrés à la conception et à l'évaluation des systèmes persuasifs car en transférant les mécanismes du jeu à d'autres

\footnotetext{
8 Terme utilisé ici pour traduire "affordances".
} 
domaines (ludification), il est possible de provoquer chez les utilisateurs, sous certaines conditions, des comportements qui ne seraient pas adoptés spontanément (faire du sport, acheter un voyage, remplir un questionnaire, etc.). Ceci a fait entrevoir de véritables "el dorado" à ceux qui pensent pouvoir maitriser la mécanique du jeu, notamment dans le secteur du commerce où la captation de l'attention du client, sa fidélisation, sont très recherchées $^{9}$. En insufflant un caractère ludique aux situations de persuasion, de nouvelles modalités d'incitation au changement peuvent être envisagées et l'on peut enrober la simple information, auparavant reçue passivement par l'individu, dans un jeu interactif entrainant le persuadé dans un « flux d'expérience optimale » (Csikszentmihalyi,1990) qui peut améliorer l'efficacité de la persuasion.

Si l'utilisation adaptée de techniques de jeu est susceptible de rendre les activités plus engageantes et plus amusantes, la mise en œuvre suppose de prendre en compte aussi bien la dynamique du jeu (c'est à dire les patterns temporels de la progression dans le jeu : rythme, rendez-vous, échéancier ...) que sa mécanique (caractéristiques rendant visibles cette progression : points, tableau de résultats, badges, niveaux, ....). De plus, l'esthétique est susceptible de déclencher une expérience émotionnelle engageante (amusement, satisfaction, surprise, fierté, ....). La convergence des travaux des deux champs pourrait avoir un fort impact sur l'efficacité des systèmes d'influence étant donné que les principes adressent des registres différents de l'expérience utilisateur:

- Dans les jeux, les principes sont plutôt orientés vers la motivation et l'engagement avec un accent fort sur les aspects émotionnels,

- Dans les systèmes persuasifs, les principes plutôt orientés vers les propriétés opérationnelles de l'interaction : performance, utilisabilité, confiance, ...

Les sections suivantes présentent un rapide tour d'horizon des travaux concernant les jeux et la persuasion en fournissant quelques pointeurs permettant au lecteur de s'y retrouver.

\subsection{Aperçu des travaux concernant le jeu}

" Homo Ludens » (Huizinga, 1938), l'expression montre assez bien que "le jeu est consubstantiel à la culture" et qu'il occupe dans les activités humaines une place importante sinon essentielle. Avec le développement des technologies numériques, le jeu a progressivement pris ses lettres de noblesse et occupé une place de plus en plus importante dans la société contemporaine. Non seulement le poids économique de ce secteur d'activité est devenu considérable (il représente $10 \%$ du chiffre d'affaires de l'industrie culturelle avec une progression de l'ordre de plus de $25 \%$ en 3 ans), mais sa pénétration dans la vie quotidienne a fait sauter les barrières entre les activités sérieuses et les activités ludiques. D'abord illustré dans le domaine pédagogique par les travaux qui ont défendu l'idée qu'il était possible d'apprendre en s'amusant, on en est venu à découvrir qu'il était aussi plus facile de vendre, de convaincre, .... Une raison de ce succès tient à ce que le jeu permet de s'échapper de la réalité : il constitue une activité séparée, libre, réglée, incertaine, improductive et fictive (Caillois, 1958). Lorsqu'il s'y engage, le joueur franchit un "cercle magique" qui le sépare du monde réel. Les frontières entre jeu et réalité sont ainsi progressivement devenues poreuses, d'abord avec le développement des jeux sérieux, puis avec l'engouement pour la gamification et maintenant avec l'émergence des jeux pervasifs ${ }^{10}$.

\footnotetext{
${ }^{9}$ Voir par exemple Badgeville (http://badgeville.com/) plateforme de gamification dédiée au B2B et visant à développer l'engagement des employés, des clients et la fidélisation vis à vis des produits.

10 Voir par exemple dans le domaine de la fiction le film "The Game" - http://lc.cx/ZSLF, les nouvelles formes de narration du Transmedia (http://fr.wikipedia.org/wiki/Transm\%C3\%A9dialit\%C3\%A9\#Notes_et_r.C3.A9f.C3.A9rences) ou encore le jeu Ingress http://en.wikipedia.org/wiki//ngress \%28game\%29 ....
} 


\subsubsection{Principaux axes de travail}

Les travaux concernant ce champ de recherche ont été développés dans trois directions principales : les études scientifiques des jeux numériques, l'ingénierie des jeux et la ludification.

Études scientifiques des jeux numériques. Les travaux scientifiques consacrés aux jeux numériques sont généralement référencés sous le terme de Game Studies. Cette discipline académique traite de l'étude critique des jeux dans la perspective des SHS et s'intéresse à leur conception, aux joueurs et à leur rôle dans la société et la culture. Les thèmes traités concernent notamment l'effet des jeux vidéo (sur les capacités cognitives, sur la violence, sur l'addiction, ...), les représentations culturelles associées, les phénomènes d'appropriation, la narration et la ludologie (science de l'interaction ludique). Pour une revue, voir Rueff $(2008)^{11}$.

Ingénierie des jeux. L'ingénierie des jeux (Game Design) est la discipline qui définit les principes de conception ; elle réunit chaque année les gourous des jeux vidéo dans les Game Developpers Conference (http://www.gdconf.com/), conférences réservées aux professionnels et hors des circuits universitaires. Le fait que ces événements ne soient pas ouverts au public académique nuit à l'établissement de consensus concernant les principes de conception et les approches restent singulières. Le savoir-faire est diffusé dans une littérature au demeurant très riche qui présente des recommandations opératoires. Voir notamment http://lc.cx/ZSL9.

Ludification. La ludification est l'application des principes du jeu à des situations qui ne sont pas ludiques: situations de travail, situations d'apprentissage, situations de résolution de problèmes ou engagement d'audience dans les situations de communication. Certains des mécanismes ludiques permettent d'influencer les comportements et viennent compléter les technologies persuasives. Ainsi, la ludification met fréquemment en œuvre un large spectre de récompenses, manipule également le divertissement et mobilise l'humour. De plus, les notions de niveaux de jeu et d'évolution inhérentes au jeu peuvent se révéler structurantes lors de la conception de dispositifs persuasifs. La ludification fait l'objet d'un buzz important ${ }^{12}$ et de critiques très vives de la part des acteurs du jeu (voir par exemple l'analyse sévère de l'ouvrage de Zichermann et Cunningham, 2011 dans http://lc.cx/ZSLt). L'approche des auteurs y est jugée purement commerciale et se limitant à l'application mécanique de techniques superficielles du jeu (principalement des «incitateurs de motivation " - motivational affordances - tels que badges, points, tableau de résultats ...) sans considérer l'importance des motivations internes et en particulier le fait que l'essence du jeu réside dans l'intérêt qu'a pour le joueur la résolution des difficultés rencontrées.

\subsubsection{Dynamique des jeux et moteur de l'engagement}

Un des aspects fascinants des jeux tient à leur capacité à engager le joueur dans un " cercle magique » (Huizinga, 1988) et à maintenir un engagement qui peut conduire à l'addiction, ce qui explique en partie l'engouement pour la ludification. Le fait qu'un individu puisse rapidement investir un temps considérable et de façon récurrente dans une activité qui provoque chez lui la satisfaction et l'envie d'y revenir est très alléchant pour le commerce en ligne. Les travaux théoriques de Caillois (1992) et de Huizinga (1988) qui analysent en détail les propriétés de la situation de jeu font encore autorité de nos jours (voir aussi Tricot, $2011^{1}$ ). On en retient ici le fait que le principe d'un jeu repose, entre autres, sur 2 aspects fondamentaux :

\footnotetext{
11 Rueff, J. (2008) Où en sont les "game studies" ? , Réseaux, 151, 139-136. http://www.cairn.info/revue-reseaux-2008-5-page-139.htm

12 Voir par exemple : http://lc.cx/ZSbZ ou http://lc.cx/ZSbo . II est même possible de suivre un MOOC sur Coursera https://www.coursera.org/courses?search=gamification.
} 
- la résolution de difficultés intéressantes pour le sujet.

- l'idée qu'il existe une expérience optimale qu'il s'agit de contrôler pour conserver le joueur dans le cercle magique.

Résolution de difficultés. Le jeu constitue une mise en situation dans laquelle le joueur est confronté à un challenge : il doit mobiliser des ressources particulières pour dépasser les difficultés auxquelles il est confronté. Dans les jeux les plus élaborés, on retrouve généralement :

- Une logique de niveaux d'apprentissage : le joueur acquiert progressivement la maîtrise du jeu et franchit des paliers modifiant son statut (novice / expert / maître).

- Des objectifs spécifiques à chaque niveau se traduisant par la mise en œuvre de principes de gratification, d'engagement, ... qui sont spécifiques et bien adaptés.

- Une mécanique de jeu constituée de l'articulation d'un sous-ensemble des leviers pouvant être utilisés pour la construction d'un jeu (voir par exemple http://gamification.org/wiki/Game Mechanics).

- Une dynamique de jeu qui définit la logique de l'interaction avec le joueur (le gameplay) et qui comporte par exemple des cycles d'engagement qui conduisent le joueur à revenir.

Le flow et la notion d'expérience optimale. La dimension émotionnelle du jeu (appelée esthétique dans le modèle MDA $^{13}$ (Mechanics-Dynamics-Aesthetics, Hunicke et al, 2004) renvoie à la notion d'expérience optimale de Csikszentmihalyi (1990) qui se manifeste par un état de bien-être issu de l'équilibre entre les compétences personnelles de l'individu et sa capacité à répondre aux demandes de la situation ${ }^{14}$ (voir aussi Asakawa, 2004). Lors d'une telle expérience, l'individu est profondément absorbé dans une activité qu'il considère comme extrêmement intéressante, comme peut l'être la résolution d'équations pour un mathématicien ou la composition pour un musicien. Cet état dynamique se caractérise par :

- un haut degré de concentration sur un champ limité de conscience (hyperfocus).

- une perte du sentiment de conscience de soi, une disparition de la distance entre le sujet et l'objet.

- la distorsion de la perception du temps.

- une rétroaction directe et immédiate (les réussites et difficultés au cours du processus sont immédiatement repérés et le comportement ajusté en conséquence).

\subsection{Aperçu des travaux concernant l'approche psychologique de la persuasion}

La figure 2 propose un éclairage des principaux champs de recherche en SHS qui fondent les principes mis en œuvre dans les technologies persuasives:

- travaux académiques de sociologie et psychologie (psychologie sociale, cognitive, communication ...), mettant notamment en évidence les biais cognitifs et comportementaux;

- travaux expérimentaux et les recherche-actions concernant la persuasion et l'engagement ;

- travaux en IHM concernant, entre autres, les aspects émotionnels de l'expérience utilisateur.

13 Dans ce modèle, souvent référencé, la composante mécanique est constituée des algorithmes et des leviers, la dynamique définit l'articulation des leviers et la progression dans le jeu, et l'esthétique renvoie au contrôle de la dimension émotionnelle.

14 http://fr.wikipedia.org/wiki/Flow_\%28psychologie\%29 


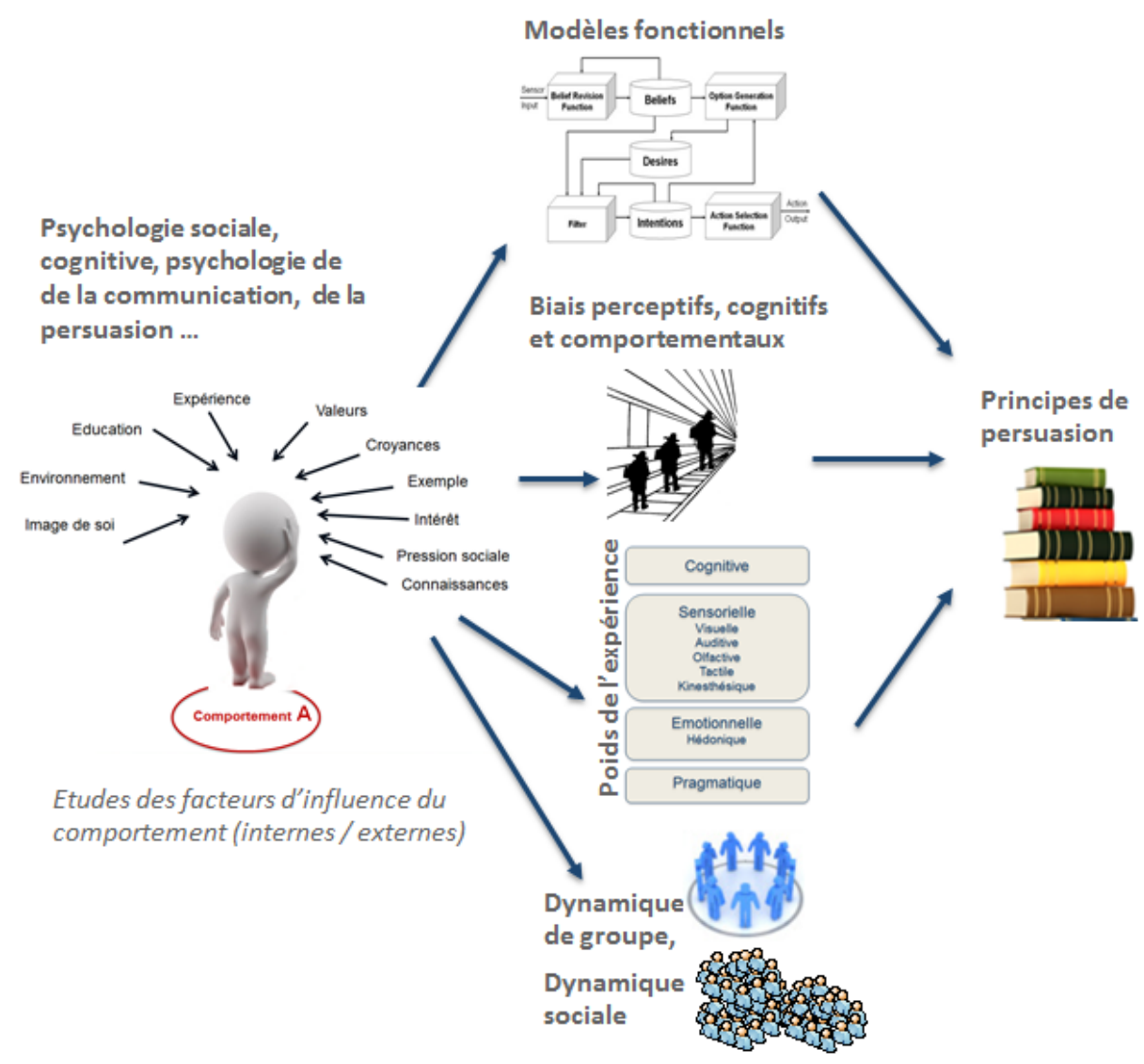

\subsubsection{Principaux axes de travail}

Les travaux théoriques en psychologie (sociale, cognitive, ...) cherchent à clarifier les relations entre les constructions mentales des individus et leurs comportements. Ils identifient les construits psychologiques, les croyances, les valeurs, les connaissances... d'un individu, les relations entre ces construits et les processus qui les mobilisent dans le cadre des cours d'actions. Ces constructions mentales sont articulées dans des modèles fonctionnels théoriques qui sont testés de façon expérimentale au regard de prédictions concernant l'adoption de comportements cibles. Les modèles élaborés sont trop nombreux et variés pour être cités ici, le lecteur intéressé en trouvera une revue dans Jackson (2005), les quelques références ci-dessous renvoient aux plus populaires dans le champ académique :

- $\quad$ recherches de l'école de Yale sur la communication persuasive (Hovland et al, 1953) cherchant à mettre en évidence les facteurs d'influence liées aux caractéristiques des messages, des locuteurs... Les expérimentations réalisées ont par exemple montré que la crédibilité d'un message dépend, entre autres, de la crédibilité de celui qui l'émet (de son niveau d'autorité hiérarchique, de son degré d'expertise dans le domaine, ....) ;

- recherche-action sur la dynamique de groupe et l'engagement (Lewin, 1947) montrant, entre autres, l'importance du caractère public des décisions pour inciter au passage à l'acte. Une décision effectuée en situation de groupe conduit l'individu à adopter par la suite des actions allant dans le même sens (effet de gel) ;

- travaux sur la dissonance cognitive (Festinger et al., 1993) illustrant les solutions mises en œuvre pour conserver une cohérence en cas de contradiction entre les faits objectifs, les comportements et/ou les attitudes. Typiquement, lorsque les actes d'un individu ne sont pas compatibles avec les valeurs qu'il défend, la réduction de la dissonance peut, entre autres, s'appuyer sur la minimisation de l'incohérence ("ce n'est pas si grave", 
"c'est la première fois"...) ou sur la justification rationnelle du comportement ("oui, mais là, ce n'est pas du tout pareil ...") ;

- $\quad$ analyse de la dynamique de groupe et développement de la sociométrie (Moreno, 1935) permettant d'identifier les rôles des différents membres d'un groupe à partir d'une quantification des échanges qu'ils ont entre eux. Ces travaux sont notamment utilisés pour renforcer la cohésion des collectifs de travail (ou parfois la réduire).

Ces travaux, complétés notamment par des études cliniques concernant la personnalité (par exemple estime de soi, auto-efficacité (Bandura, 1969)) ou centrées sur la logique de la communication (Bandler et Grinder, 1982; Watzlawick, 1967) ont permis d'identifier des principes d'influence et d'élaborer des techniques d'accompagnement du changement qui s'appuient sur les mécanismes psychologiques identifiés. Ces techniques ont été appliquées dans des domaines aussi divers que la communication, les organisations, le développement personnel, la thérapie comportementale,... et sont parfois structurées en véritables méthodologies. C'est le cas par exemple de Indimark ${ }^{\mathrm{TM}}$ (Brög et al. 2002), méthode de marketing individualisé destinée à changer les comportements de mobilité. Le dispositif mis en place consiste à sélectionner une population cible à partir d'un profilage. Un contact direct est ensuite établi pour poser un diagnostic personnalisé concernant les modes de transport utilisés au regard de ceux qui sont disponibles. Des alternatives de transport sont proposées en identifiant les ressources de mobilité les plus adaptées. Après le choix d'alternatives, un suivi régulier de leur mise en œuvre est effectué et, au besoin, les renforcements nécessaires à la pérennité du changement sont fournis. Mis en œuvre dans différents pays, l'efficacité de ce dispositif a été évaluée de façon systématique (Coester et al. 2002).

\subsubsection{Diversité des points de vue concernant les moteurs du changement}

Un examen des différentes approches du changement montre qu'elles reposent sur des modèles incompatibles dans lesquels les moteurs de l'action sont sensiblement différents. Selon que l'on considère par exemple le sujet cible comme un acteur rationnel ou au contraire comme ayant une rationalité limitée, les déterminants du changement sont recherchés dans la structuration de l'argumentation ou placés dans d'autres registres : celui des émotions (Damasio, 1995), dans la prise en compte des valeurs, (Schwarzt, 2006), dans l'analyse de la motivation (Fenouillet, 2012) ... Pour d'autres auteurs, ce qui fait agir le sujet c'est la volonté de réduire des tensions internes que ressentirait celui que l'on veut persuader. Ainsi, la communication engageante (Joule et Beauvois, 1999) s'appuie entre autres sur la notion de dissonance cognitive (Festinger et al. 1993) souvent mise à contribution dans le champ de la persuasion. D'autres modèles de résolution de tension s'intéressent à l'image de soi, à la peur de perdre ou à l'anxiété liée à la prise de décision.

\subsubsection{Principes de persuasion}

Fogg a ouvert le champ de la "captologie " en définissant les outils de persuasion technologique comme étant des dispositifs interactifs conçus pour changer les attitudes ou les comportements (voire les deux) en rendant ces changements plus faciles à atteindre. Sur cette base, il a identifié 7 types d'outils technologiques :

- Les outils de réduction de la complexité, utilisés pour rendre les choses plus simples.

- Les outils de structuration (tunneling) qui persuadent par un guidage pas à pas.

- Ceux qui, basés sur la personnalisation (customization), fournissent des informations adaptées à chaque utilisateur.

- Ceux qui fournissent des conseils et des suggestions.

- D'autres outils mettent les utilisateurs en situation d'autocontrôle en leur fournissant des ressources pour évaluer leurs propres performances.

- Le changement peut aussi être facilité grâce à des outils de surveillance qui diffusent à autrui les données qui ont été acquises.

- Enfin l'implémentation de techniques de conditionnement opérant (récompense / pénalité) renforcent l'adoption d'un comportement cible. 
Cette typologie a été complétée par un ensemble de constructions théoriques visant à structurer les travaux de captologie parmi lesquels on peut citer entre autres :

- les trois rôles fonctionnels (outils, média ou acteur social) que peuvent jouer les ordinateurs au cours des interactions (Fogg, 1998) ;

- les différents stades d'engagement lors des participations en ligne (découverte, engagement superficiel, engagement profond - Fogg et Eckles, 2007) ;

- le Fogg's Behavior Model (FBM, appelé aussi MAT pour Motivation, Ability, Triggers), un modèle structurant les relations entre les principaux déterminants du changement de comportement et censé faire la synthèse des travaux de psychologie (Fogg, 2009) ;

- Le Behavior Wizard, une typologie des classes de situations de persuasion (Fogg et Hreha, 2010) établie en fonction des objectifs poursuivis (cf. http://www.behaviorwizard.org/wp/) .

Outre le catalogue de principes de persuasion initialement établi par Fogg (2003), la littérature et les sites en ligne présentent une diversité de principes d'influence. Sans en établir un recensement exhaustif, on peut citer parmi les plus fréquemment référencés :

- les principes proposés par Cialdini (2004). Ils sont dérivés d'études de terrain concernent les manipulations mises en œuvre lors des interactions en face à face. L'auteur, professeur de psychologie sociale, les a identifiés en occupant divers postes (vendeur de voiture d'occasion, démarcheur pour des organisations caritatives, télémarketing). On y retrouve un florilège de techniques très efficaces pour faire adopter le comportement spécifique telles que la rareté ("c'est le dernier et je n'en aurai plus"), la pression sociale ("Tout le monde en a ! Pas vous ?"), la réciprocité ("prenez, c'est gratuit" - le cadeau crée la dette), l'autorité ("je ne suis pas un vendeur, je suis missionné par la direction générale pour animer temporairement l'équipe de vente"), la sympathie ("il me semble que nous nous sommes déjà rencontrés, non ?") etc....

- le modèle Persuasive System Design (PSD) proposé par Oinas-Kukkonen et Harjumaa (2008a, 2008b, 2009) constitue un important effort de structuration des principes initiaux de Fogg. Ces principes sont complétés et répartis dans 4 classes selon qu'ils facilitent la tâche principale, qu'ils relèvent du dialogue, qu'ils renforcent la crédibilité ou qu'ils s'appuient sur la dimension sociale de la persuasion. Ce modèle a servi de cadre d'analyse pour rendre compte de la qualité persuasive d'un dispositif de biofeedback (Harjumaa et al., 2009).

- Plus récemment, Némery (2012) a abordé le champ de la persuasion dans une perspective d'ergonomie des $\mathrm{IHM}^{15}$. L'auteur propose une "grille de critères ergonomiques pour l'appréciation de la persuasion technologique" organisée selon un modèle hiérarchique. Deux classes principales distinguent les aspects statiques et les aspects dynamiques, dans lesquels un ensemble de dimensions regroupe chacune plusieurs critères élémentaires. Par exemple, les aspects statiques sont organisés selon 4 dimensions (crédibilité, privacité, personnalisation et attractivité), elles-mêmes décomposées en 12 critères élémentaires. Les aspects dynamiques sont organisés selon un processus qui accroit le niveau de contrôle sur l'utilisateur : une première phase de sollicitation s'appuie sur la suggestion et la mise en curiosité, elle est suivie d'un accompagnement initial censé pousser ensuite à l'engagement de l'utilisateur et finalement à une phase dans laquelle une emprise peut conduire à des comportements relevant de l'addiction. L'auteur s'appuie sur les travaux des précurseurs (Fogg, 2003, Oinas-Kukkonen et Harjumaa, 2009; Lockton et al. 2010) en faisant l'effort d'établir les références aux travaux plus théoriques sous-jacents. La catégorisation des principes a fait l'objet d'une validation expérimentale avec des experts d'ergonomie des IHM (Némery et al., 2011). Une mise à l'épreuve a de plus été effectuée en appliquant des

\footnotetext{
15 Voir aussi Némery et Brangier (2014).
} 
principes d'ergonomie incitative pour augmenter le taux de réponse d'un sondage en ligne effectué tous les ans dans une entreprise.

- $\quad$ On notera également le développement d'un "marché de la persuasion" avec des offres de formation de la part d'acteurs opérationnels tels que $N N / g^{16}$ ou Human Factors International ${ }^{17}$ qui propose des heuristiques de marketing interactif basées sur le triplet PET (Persuasion, Emotion, Trust, ${ }^{18}$ ). Les technologies numériques (bigdata, linguistique computationnelle ...) facilitent l'émergence de services d'ingénierie persuasive tels que ceux de Persado (http://www.persado.com/). Cette société, créée en 2012, propose à ses clients une technologie permettant d'élaborer le message commercial le plus efficace pour générer des conversions.

La multitude de travaux conduits depuis des décennies dans les champs de recherche sur le jeu et la persuasion évoqués ci-dessus a fait émerger les notions et les concepts sur lesquels s'appuie la boite à outils actuelle des SLP. Les diverses implémentations réalisées jusqu'à présent n'ont conduit ni au consensus concernant les déterminants de l'influence et du changement ni à l'établissement d'un catalogue définitif de principes de persuasion et de ludification : de ce fait, les défis à relever pour en faire des dispositifs efficients restent importants. Quelques-uns d'entre eux sont discutés dans la section suivante.

\section{DEFIS DE LA CONCEPTION ET DE L'EVALUATION DE SLP POUR LA CONSOMMATION DURABLE}

Le concepteur d'un système ludo-persuasif dispose d'une boîte à outils comportant, entre autres, un ensemble de principes de jeu et de persuasion. Le dispositif est conçu pour atteindre des objectifs de changement bien déterminé (comportement ou attitude cible) et lorsque l'artefact est mis à disposition d'une population donnée (population cible), son utilisation régulière et le développement des usages sont censés conduire à l'adoption du comportement visé et dans le meilleur des cas à sa pérennité. Cette problématique générale est représentée dans la figure ci-dessous :

Figure 3 : Problématique SLP - conduire à l'adoption d'un comportement par des interactions

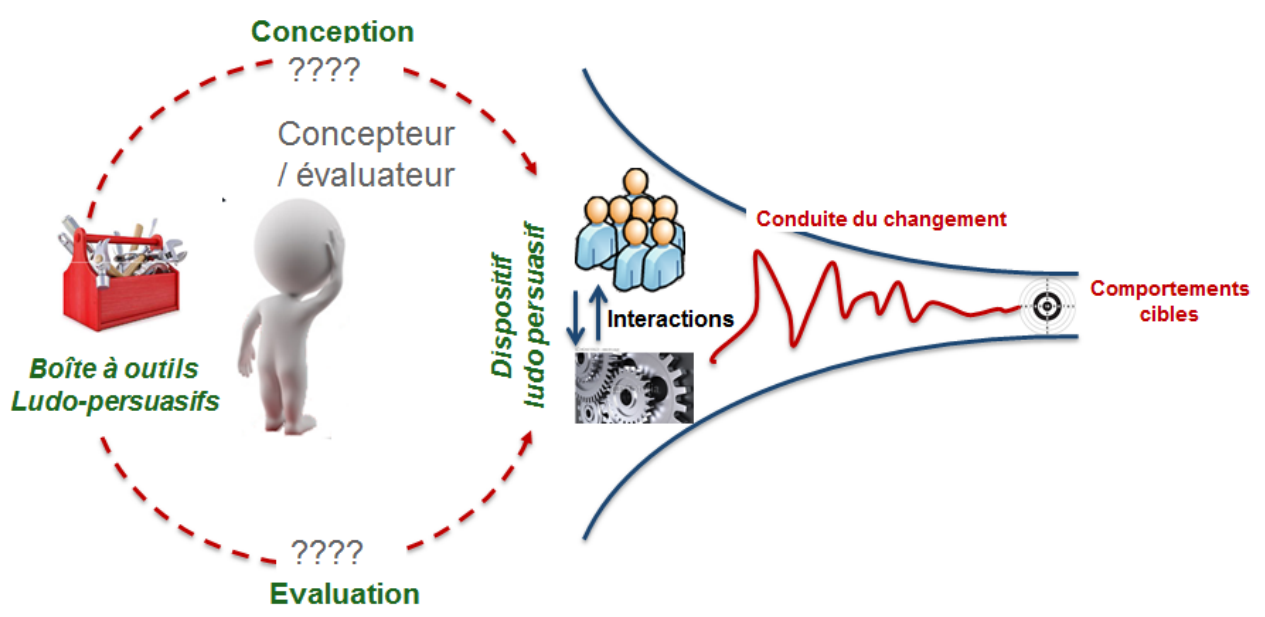

Pour élémentaire qu'elle soit cette présentation pointe quelques unes des exigences de la conception. Le concepteur doit prendre un ensemble de décisions stratégiques et

\footnotetext{
${ }^{16}$ Nielsen Norman Group : http://www.nngroup.com/courses/credibility-and-persuasive-web-design/

17 http://www.humanfactors.com/home/usability.asp

${ }^{18}$ http://fr.slideshare.net/QuiffBoy/pet-designing-for-persuasion-emotion-and-trust
} 
tactiques, concernant le choix et l'articulation des leviers de jeu et de persuasion permettant d'éviter les rebonds ou l'abandon en cours d'utilisation. II doit inciter à effectuer des actions élémentaires qui orientent et engagent vers le comportement cible, donnant envie de revenir utiliser le système. II doit :

- en premier lieu caractériser précisément les objectifs de changement à atteindre (cahier des charges) ;

- définir la stratégie de mise en œuvre compte tenu des caractéristiques des populations ;

- sélectionner les principes les plus adaptés aux objectifs du cahier des charges puis les articuler de façon cohérente pour atteindre ces objectifs ;

- et finalement les implémenter sous la forme la plus adéquate.

Les options prises en cours de conception doivent aussi être validées, notamment du point de vue du degré d'efficacité des solutions utilisées, de la qualité des implémentations et du respect des exigences usuelles d'utilisabilité. Outre ces exigences, dans un SLP, d'autres aspects relatifs à la "récréativité ${ }^{19}$, (dynamique de jeu - gameplay) et la " persuabilité » des systèmes doivent aussi être considérés. Les principes spécifiques de ludification et de persuasion doivent être choisis et articulés de sorte à établir les parcours de persuasion les plus efficients tout en maintenant l'intérêt des utilisateurs. Ces exigences diverses suggèrent que les obstacles à dépasser sont nombreux et, en fait, les défis sont d'autant plus difficiles à remporter qu'il n'existe pas à l'heure actuelle de directives (guidelines), de guide pratique, de manuel ... facilitant la sélection, l'articulation et l'implémentation des principes à mettre en œuvre. Dans quelle mesure les techniques développées pour l'utilisabilité des IHM et les outils disponibles sont-ils directement transférables aux SLP ? Quels aménagements doivent être effectués, quelles techniques complémentaires doivent être développées ... ? Cette section cherche à poser quelques-uns des jalons qui pourraient permettre d'apporter des éléments de réponse à ces questions. Les cinq défis discutés cidessous sont cadrés dans le contexte de la consommation durable, c'est à dire en considérant des dispositifs dont la finalité est de conduire ses utilisateurs à adopter de façon pérenne des éco-gestes. Ces défis sont représentés dans la figure ci-dessous.

Figure 4 : 5 défis de conception et d'évaluation d'un SLP

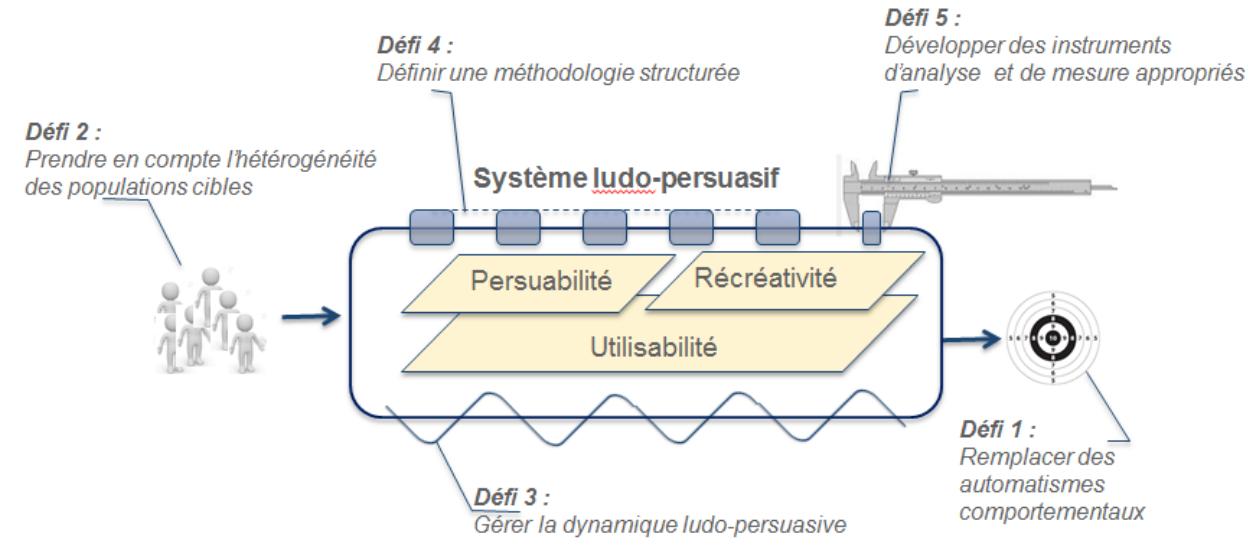

\subsection{Défi 1 : remplacer des automatismes comportementaux}

La plupart des individus ont pu expérimenter les difficultés que l'on a à changer son comportement et ce, bien que l'on ait des motivations suffisamment fortes pour être passé à

\footnotetext{
${ }^{19}$ Au Québec, le terme français "jouabilité" est utilisé pour traduire gameplay. On lui préfère ici celui de récréativité du fait que " jouabilité " est un terme aussi utilisé pour qualifier l'utilisabilité des méthodes d'interaction et des dispositifs de contrôle-commande utilisés dans les jeux (panel d'actions, combinaison des actions, joystick, manettes, ..)
} 
l'acte : abandon plus ou moins rapide de régime alimentaire, d'activités sportives, tentatives successives pour arrêter de fumer, abandon progressif des décisions prises en début d'année, etc. Ils ont aussi pu expérimenter la facilité avec laquelle ils adoptent de nouveaux comportements, quand par exemple des nouveaux dispositifs technologiques sont mis sur le marché (Smartphones, ...) ou que des services à forte valeur ajoutée deviennent disponibles en ligne (cf. par exemple Recherche Google, Twitter, Pinterest, Picasa ...).

\subsubsection{Une difficulté bien connue}

Ainsi, autant il semble facile d'adopter de nouveaux comportements, autant il est difficile de se débarrasser des anciens. En l'occurrence, une des difficultés spécifiques du changement des modes de consommation tient à ce qu'il est nécessaire de remplacer des routines quotidiennes, des habitudes très structurées par de nouvelles modalités d'actions qui, elles-mêmes, doivent être pérennisées. Ces habitudes sont des "scripts d'actions" très structurés dont le déclenchement est automatique et dont la difficulté d'abandon est généralement illustrée par l'adage bien connu du « naturel qui revient au galop » ou par les métaphores de gestion de piles (FILO : first in, last out et LIFO : last in, first out).

Ces constats illustrent la diversité des situations de changement comportementaux que l'on peut avoir à traiter et les niveaux de difficultés qui peuvent leur être associés. Ils complètent la typologie proposée par Fogg et Hreha (2010) déjà évoquée ci-dessus, en montrant que les objectifs élémentaires identifiés par ces auteurs peuvent être combinés en des objectifs de changement plus complexes (en l'occurrence suppression d'un comportement pour en adopter un nouveau). Remplacer de mauvaises habitudes par d'autres qui le sont moins, n'est pas si facile. Pour y parvenir, Eyal (2014) a récemment proposé une démarche structurée dans laquelle des heuristiques "d'hameçonnage " sont susceptibles de créer chez les utilisateurs des réflexes d'utilisation des produits. Cette piste, qui pourrait être intéressante pour les SLP est rapidement présentée ci-dessous.

\subsubsection{Un modèle de création d'habitude}

Dans les systèmes persuasifs, le poids des mauvaises habitudes est souvent négligé et généralement ni le "détricotage » des automatismes, ni l'implémentation de nouveaux comportements ne sont soutenus par des aménagements spécifiques. La "reprogrammation" des chaînes de réponse est une opération longue et subtile et il faut plus de 21 jours pour changer une habitude ${ }^{20}$. Conçu avec l'objectif explicite d'augmenter la "durée de vie du consommateur"21, le modèle "crochet" (hook) d'Eyal (2014) considère que, pour créer I'habitude d'utiliser un service en ligne, il ne suffit pas d'augmenter la fréquence des cycles d'interactions. II faut de plus transformer la motivation basée sur une accroche initiale externe en une motivation interne. La stratégie utilisée pour cela s'appuie sur des renforcements positifs et sur une dynamique des récompenses dont la variabilité entretient une surprise et un intérêt suffisant pour inciter l'utilisateur à revenir. Le dernier composant du modèle permettant de capter le consommateur est de le rendre dépendant d'un service en ouvrant celui-ci et en mettant l'utilisateur en capacité de créer de la valeur. Par exemple, le stockage et la réutilisation dans d'autres contextes de données acquises grâce au service crée une dépendance, de plus, si l'abandon du service détermine la perte de ressources utiles à l'usager, cela dévalorise complètement les investissements qu'il a pu effectuer précédemment et il a du mal à s'y résoudre.

\footnotetext{
${ }^{20}$ Cette durée communément admise a été mise en question par une étude systématique de Lally et al. (2010) conduite auprès de 96 volontaires sur 12 semaines montrant qu'une durée moyenne de 66 jours est nécessaire pour l'acquisition de nouveaux comportements. La dispersion très importante montre aussi qu'il y a une grande variabilité interindividuelle.

${ }^{21}$ Définie par rapport au montant financier prélevé sur un client avant qu'il ne parte à la concurrence, qu'il arrête d'utiliser le produit ou qu'il ne meure (sic).
} 
La figure 5 représente le "module" persuasif élémentaire susceptible de favoriser l'installation de nouvelles habitudes.

Figure 5 : Logique du modèle "crochet" (Eyal, 2014)

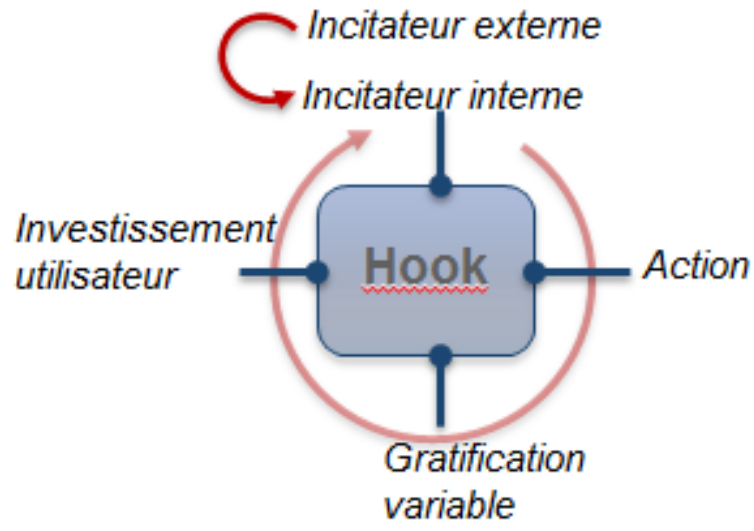

\subsubsection{Nécessité de données ethnographiques}

Dans quelle mesure ce modèle peut-il être utilisé pour inciter à adopter des éco-gestes ? Si une validation de ce modèle montre qu'il se révèle effectivement efficace pour faire évoluer l'utilisation d'un système interactif vers une habitude compulsive, il restera à l'instancier dans un contexte d'acquisition d'éco-gestes et à déterminer dans quelle mesure des routines d'utilisation d'un dispositif peuvent suffire à déterminer un changement de comportement. II semble aller de soi, à la réflexion, qu'une " mécanique d'interaction " ne peut trouver son efficacité que si elle s'appuie en préalable sur une compréhension des contextes dans lesquels les routines à supprimer sont mises en œuvre. II faut identifier leurs déclencheurs et comprendre les éventuels bénéfices secondaires associés aux comportements non désirés... Un recueil de données ethnographiques permettant d'acquérir des éléments de cadrage de la stratégie de persuasion et de conduite du changement est alors une première piste vers l'amélioration de l'efficacité des SLP.

\subsection{Défi 2 : prendre en compte l'hétérogénéité des populations cibles}

Les travaux conduits en ergonomie des IHM ont montré à quel point la connaissance des utilisateurs et l'élaboration de profils pouvaient améliorer notablement les performances des systèmes hommes-machines et la qualité de l'expérience utilisateur : la prise en compte du niveau de connaissances, de la logique d'activité, de l'expérience d'usage, .... permettent d'adapter les contenus, les modalités d'interaction, d'anticiper les besoins et de les satisfaire au mieux (Levieux, 2011). Ces connaissances sont regroupées dans des modèles utilisateurs en un ensemble d'informations permettant de prédire le comportement en fonction de stimulations qui sont fournies. Ces modèles sont plus ou moins sophistiqués, ils peuvent par exemple dans les jeux, s'appuyer sur les 5 traits de personnalité identifiés par Golberg $(1990)^{22}$ ou sur une typologie permettant de caractériser les centres d'intérêt des utilisateurs, comme par exemple celles proposées pour les joueurs par Bartle $(1996,2005)$, un pionnier des jeux en ligne multi-joueurs.

La question de savoir quelles connaissances sur les utilisateurs pourraient contribuer à améliorer les performances d'un SLP pour la consommation durable ouvre un champ de travail. En première analyse, deux aspects semblent particulièrement importants :

\footnotetext{
22 Les Big Five : distinction établie entre 5 traits de personnalité psychologique, à partir d'une analyse factorielle. extraversion, névrosisme, agréabilité, conscience et ouverture à l'expérience.
} 
- la distance à la cible ;

- la sensibilité différenciée des individus aux principes de persuasion.

La discussion de ces deux aspects conduit à envisager l'utilité de personas pour les SLP, piste discutée section 4.2.2.

\subsubsection{Différences interindividuelles : distance à la cible}

La population ciblée par un dispositif de développement durable est généralement hétérogène du point de vue des attitudes vis-à-vis de cette problématique, du niveau d'engagement, des actions déjà entreprises dans ce domaine. On utilise ici le terme de "distance à la cible" pour caractériser ces différences entre les individus. Les objectifs d'un SLP doivent être adaptés en fonction des caractéristiques de la population cible : selon qu'il s'agit de sensibiliser les individus, de les aider à passer à l'action, de les engager dans une démarche ou de pérenniser leurs comportements, les exigences à satisfaire, la finalité du dispositif et les principes de jeu et de persuasion à mettre en œuvre doivent être sensiblement différents. Par exemple :

- les jeux sérieux sont fréquemment utilisés pour la sensibilisation aux économies d'énergie et on en trouve en ligne de nombreuses illustrations (ElectroCity ${ }^{23}$, 2020 Energy $^{24}$, Energuy ${ }^{25}$, lachezprise ${ }^{26}$; voir aussi la littérature scientifique relative à Poweragent (Bang et al, 2007) ou à PowerHouse (Bang et al, 2006)).

- Les incitations destinées à faciliter le passage à l'acte doivent s'appuyer sur les modèles qui identifient les conditions à satisfaire pour qu'un comportement donné soit effectué. Par exemple, le modèle MOA (Motivation, Opportunity, Abilities) de Maclnnis and Jaworski (1989) ${ }^{27}$ est un de ceux qui est le plus souvent référencé. II suggère que la mise en œuvre d'un comportement donné nécessite de satisfaire simultanément ces trois conditions. Tout au long d'une conception, il faut renforcer la motivation par des principes adaptés, et mettre les utilisateurs en capacité d'agir en utilisant des déclencheurs adéquats.

- La pérennisation nécessite l'établissement de diagnostic concernant les difficultés rencontrées et une assistance adaptée pour les dépasser. L'implémentation de principes de jeu et/ou de persuasion facilitant l'entraide entre les individus peut alors devenir opportune.

\subsubsection{Sensibilité différenciée aux principes de persuasion}

Dans un travail de thèse très intéressant, Kaptein (2012) aborde la question d'une sensibilité différentielle des individus aux principes de persuasion. Développés dans une perspective de "persuasion profiling" (Kaptein et al , 2010) ces travaux montrent que les individus ont des réactions différentes aux différents leviers utilisés pour persuader : certains sont insensibles à l'autorité, d'autres réagissent à la pénurie,... et ces réactions sont stables. De plus, une expérimentation a confirmé que les personnes suivent plus fréquemment des

\footnotetext{
${ }^{23} \mathrm{http://www.electrocity.co.nz/} \mathrm{.} \mathrm{I'utilisateur} \mathrm{est} \mathrm{en} \mathrm{charge} \mathrm{d'une} \mathrm{petite} \mathrm{ville,} \mathrm{dont} \mathrm{il} \mathrm{doit} \mathrm{développer} \mathrm{les} \mathrm{activités,} \mathrm{tout} \mathrm{en} \mathrm{prenant} \mathrm{garde} \mathrm{à}$ entretenir son approvisionnement en énergie et en maintenant l'équilibre environnemental.

24 http://www.2020energy.eu/game

${ }^{25}$ http://efficaciteenergetique.mrn.gouv.qc.ca/energuy/jeu

${ }^{26}$ http://lachezprise.qc.cal

${ }^{27}$ Ce modèle est à l'origine du modèle MAT - Motivation, Ability, Trigger - présenté par Fogg (2009) et est présenté dans Rothschild (1999).
} 
incitations lorsque celles-ci sont basées sur des principes auxquels ils sont sensibles ${ }^{28}$. II est ainsi possible d'envisager la définition de profils de persuasion prenant en compte ces différences de sensibilité et dont pourrait dépendre le choix des principes implémentés dans un SLP.

\subsection{Défi 3 : gérer la dynamique ludo-persuasive}

La gestion de la dynamique de la persuasion suppose que le système ludo-persuasif soit en mesure de gérer un ensemble de processus concernant notamment 4 aspects discutés ci-dessous :

- $\quad$ L'engagement initial des individus dans un processus de changement.

- Le maintien de l'engagement pendant ce processus.

- La conduite du changement comportemental.

- Le monitoring du changement.

\subsubsection{Engagement initial dans un processus de changement}

L'engagement dans un jeu s'appuie sur des motivations qui, selon Bartle (1996) définissent des profils bien différenciés ("tueur", "explorateur", "accomplisseur", "socialiseur"). La motivation d'un accomplisseur est par exemple d'acquérir la maitrise des difficultés (perceptives, logiques ou physiques) introduites dans le jeu alors qu'un explorateur est là pour découvrir des univers, un territoire ou un trésor. Chacun choisit le type de jeu qui lui convient et s'y engage ou non en fonction du plaisir qu'il y prend et de la qualité des implémentations.

L'utilisation d'un système persuasif est déterminée par la volonté de changer : parvenir à maigrir, à faire du sport, à faire des économies d'énergie ... et l'aide fournie par le dispositif doit faciliter la réalisation d'un effort. Le risque est grand d'un abandon rapide et pour réduire ce risque, dans les SLP, l'accent est davantage placé sur la gestion de l'interaction que sur des modèles de progression vers la cible : le découpage en paliers est construit avec l'objectif d'établir une relation avec l'utilisateur et de la préserver. C'est par exemple le cas des 4 étapes de dynamique proposée par (Nemery, 2012) pour l'ergonomie incitative : sollicitation, accompagnement initial, engagement et emprise. L'amorçage initial est déterminé par des techniques de suggestion, de mise en curiosité, complétée ensuite par des incitations à l'action, un guidage et des encouragements. Un des défis à relever lors de la conception des SLP est donc de montrer aux utilisateurs, et ce quelle que soit leur distance au comportement cible (ou phase MTT pour reprendre le modèle transthéorique cité plus haut), ce que le dispositif peut leur apporter immédiatement, par exemple en affichant des qualité hédoniques (ex. graphisme) ou des éléments surfant sur la motivation externe de l'utilisateur (réduction, cadeaux) qui serviront d'appât initial.

\subsubsection{Maintien de l'engagement: cycle de vie d'un SLP}

Dans les jeux, la motivation initiale du joueur est maintenue par l'organisation de niveaux de difficultés et le sentiment d'une expérience optimale. Le cycle de vie d'un jeu est lié à la capacité du joueur à passer d'un palier à un autre, la progression supposant d'acquérir petit à petit la maitrise des difficultés rencontrées. Avec l'implémentation d'un modèle de persuasion comportant des étapes de progression vers la cible, à chaque palier, l'utilisateur peut trouver ses limites et rester au même niveau ou abandonner.

\footnotetext{
28 Dans une expérimentation, différents types de principes persuasifs sont utilisés pour inciter les gens à prendre les escaliers. Le traçage des comportements à partir des puces bluetooth de leur mobile montre une sensibilité différentielle aux messages : les gens réagissent plus souvent aux messages correspondant à leur profil.
} 


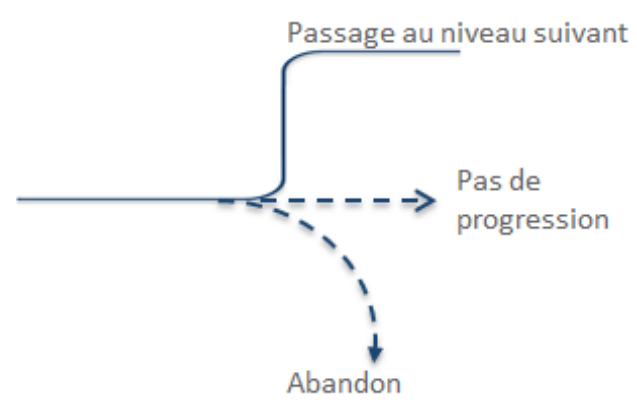

Dans le modèle Hook d'implémentation d'habitude présenté plus haut, le cycle de vie est prolongé d'une part par la variabilité des récompenses fournies qui conserve la curiosité et l'intérêt et d'autre part par le coût de l'abandon du fait des investissements déjà réalisés. Ces techniques de maintien de l'engagement restent superficielles et encore assez éloignées d'une véritable conduite du changement : celle-ci supposerait que des principes spécifiques soient associés à chacune des étapes de progression mais, le modèle de progression le plus adéquat reste à identifier et les associations entre les principes et les étapes devront ensuite être validées empiriquement.

\subsubsection{Progression : quel modèle de conduite du changement comportemental ?}

Un SLP peut être ciblé sur une étape particulière du processus de changement (sensibilisation, passage à l'acte ....) mais peut aussi offrir un accompagnement complet tout au long de ce processus, comme ce peut être le cas par exemple dans un système dédié à l'amélioration des comportements alimentaires. La conception d'un tel dispositif suppose d'avoir une compréhension précise de l'ordonnancement des étapes et de ce qui s'y passe. De ce point de vue, le Modèle Transthéorique (appelé ci-dessous MTT) proposé par Prochaska et DiClemente (2005) est une référence incontournable. Ce modèle rend compte de la dynamique des attitudes d'un individu depuis le déni vis à vis de l'existence d'un problème jusqu'au changement de comportement et à l'installation de nouvelles habitudes. Élaboré dans un contexte de traitement des addictions, il a été utilisé dans différents contextes, y compris dans le contexte écologique (cf. Boutaud, 2009). Le modèle identifie 6 phases successives dans lesquelles des modalités d'accompagnement particulières doivent être mises en œuvre pour éviter les retours en arrière (rechutes) et pérenniser le changement. II est caractérisé par un développement dans la durée et, bien que critiqué (West, 2005; Csillik, 2009), il constitue un outil structurant car il entre dans le détail, contrairement aux modèles plus classiques qui s'en tiennent à trois phases macroscopiques: "incitation à la participation, embarquement et maintien de l'embarquement ». Un aspect intéressant pour les SLP concerne l'articulation des principes ludo-persuasifs aux différents stades du modèle :

- Chaque stade définit les objectifs spécifiques qu'un SLP devrait poursuivre à ce stade.

- Des ressources spécifiques doivent être mobilisées en fonction du stade auquel se trouve un individu donné pour le conduire au stade suivant.

- Des difficultés spécifiques apparaissent à chacun des stades et des solutions adaptées doivent être mises en œuvre.

Les 6 étapes du modèle MTT sont représentées dans la figure 7. À chaque étape, des ressources de persuasion et de ludification spécifiques doivent être mobilisées (argumentation, incitateurs, ....) pour traiter les difficultés que rencontre l'utilisateur du dispositif et satisfaire ses besoins et ses attentes. 


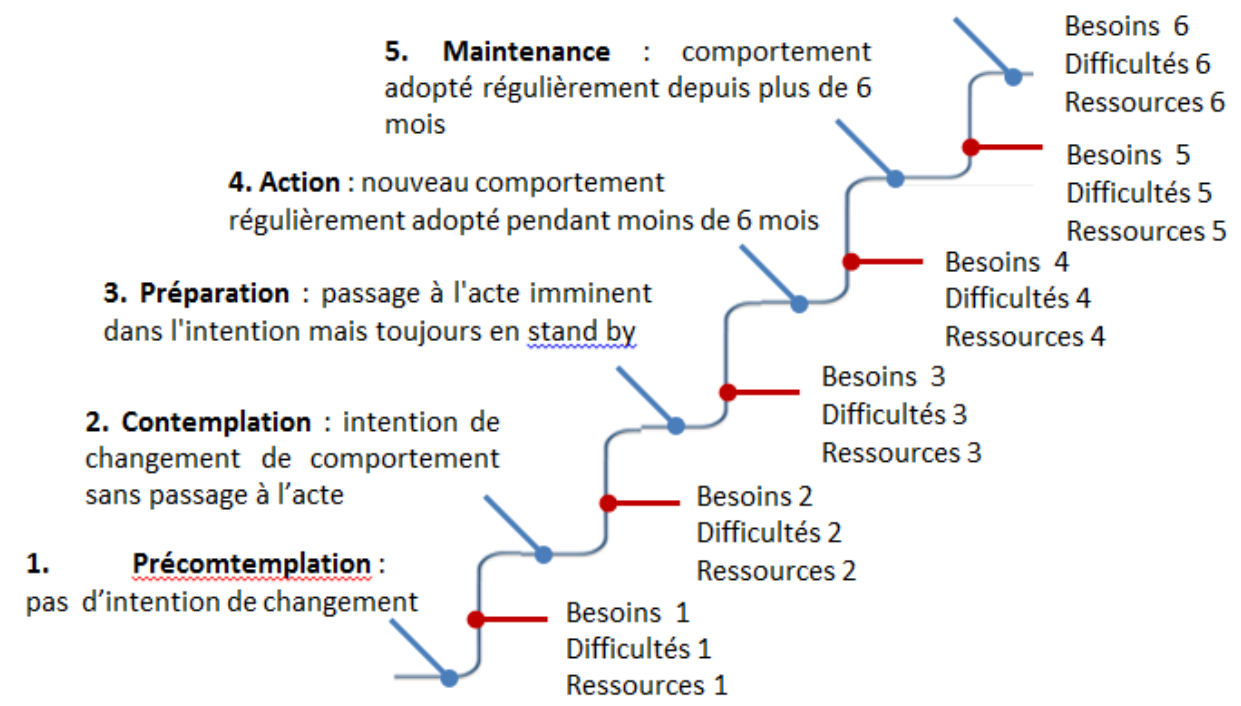

La pertinence de ce modèle dans le contexte du développement durable reste à valider. Des études conduites en situation naturelle, telles que celles de Lockton et al. (2013) peuvent contribuer à fournir des éléments de réponse. Quel que soit le modèle de progression vers la cible, l'identification des difficultés spécifiques rencontrées à chacune de ces étapes lors d'une démarche d'adoption d'éco-gestes serait de toute évidence d'un grand secours pour améliorer l'efficacité d'un SLP. De la même façon, être en mesure de qualifier les principes ludo-persuasifs en fonction de leur pertinence à tel ou tel stade de la conduite du changement serait aussi une avancée importante.

\subsubsection{Monitoring du changement : tracer la dynamique d'évolution}

Des individus différents réagissent de façon différente aux techniques de persuasion et lors de l'utilisation d'un dispositif, ils suivent des parcours différents. Déclencher au moment opportun (kairos) un guidage, une suggestion, un conseil améliore l'efficacité d'un dispositif persuasif, mais cela suppose une compréhension fine des événements qui se produisent au cours des interactions, et la gestion dynamique des incitateurs pour orienter la suite d'une transaction suppose des capacités d'inférence élevées. La supervision des parcours des utilisateurs au cours d'une session d'utilisation est un premier niveau de monitoring du changement qui peut s'appuyer sur les logs. Ceux-ci, associés à des données de profils des utilisateurs, sont des données exploitables pour assurer ce suivi à partir des navigations qui, en première analyse, permettraient :

- de tracer les difficultés rencontrées afin d'ajuster la « trajectoire de persuasion »;

- d'identifier les «patrons de persuasion » les plus efficaces ;

- de qualifier la sensibilité individuelle aux principes à travers des abandons systématiques de certains utilisateurs dans des contextes identiques.

Cependant, si le changement comportemental se produit par des avancées progressives vers le comportement cible, il est aussi nécessaire de pouvoir qualifier l'état d'avancement des individus, le stade auquel il se trouve. Dans la littérature traitant des technologies persuasives, les distinctions classiques du type «novice/expert » ne sont, pour l'instant, pas évoquées, mais elles sont présentes dans le domaine de la gamification du fait de l'existence de niveaux de jeu. Par exemple pour Amy Jo Kim, spécialiste des communautés en ligne et personnalité influente du Game Design, la maîtrise d'un jeu passe par 3 niveaux d'habiletés (novice, expert, maître) qui structurent des cycles de vie du jeu, le passage à un niveau 
donnant accès à de nouvelles possibilités, ce qui incite à franchir les obstacles pour pouvoir continuer à jouer.

Pour ce qui est de la persuasion, si l'on s'en tient à la maîtrise de l'interaction, les étapes successives d'avancée vers la cible déterminent une évolution des besoins de l'utilisateur en fonction de la fréquence des interactions avec le dispositif :

- Les novices doivent être accueillis (on boarding) ce qui suppose un traitement spécifique (mots clés : accueil, buts, progression, récompense atteignable) ;

- Les experts ont besoin de nouveauté (mots clés : contenu, actions, relations) et de prise de contrôle (mots clés : statut, personnalisation, outils facilitateurs);

- La maitrise donne accès à des privilèges très recherchés (mots clés : accès exclusif, activités additionnelles, déblocage, privilèges).

Le tableau ci-dessous donne un aperçu des différents objets d'analyse permettant d'identifier la dynamique de l'évolution des individus vers le comportement cible :

Tableau 1 : Objet d'analyse des trajectoires vers le comportement cible

\begin{tabular}{|l|l|l|}
\hline Objet d'analyse & Définition & Marqueurs \\
\hline $\begin{array}{l}\text { Chronogramme de } \\
\text { la route vers le } \\
\text { changement suivie } \\
\text { par l'utilisateur }\end{array}$ & $\begin{array}{l}\text { Succession plus ou moins } \\
\text { chaotique (retours arrières, } \\
\text { abandons, reprises ...) des } \\
\text { étapes suivies par un individu } \\
\text { dans le cadre d'un modèle de } \\
\text { changement (type MTT) }\end{array}$ & $\begin{array}{l}\text { Marqueurs spécifiques d'une } \\
\text { étape } \\
\text { Marqueurs de transition } \\
\text { Datation des logs ... }\end{array}$ \\
\hline $\begin{array}{l}\text { Modification de la } \\
\text { sensibilité aux } \\
\text { principes }\end{array}$ & Perméabilité acquise & Marqueur de réactivité à définir \\
\hline $\begin{array}{l}\text { Maîtrise de } \\
\text { l'interaction }\end{array}$ & $\begin{array}{l}\text { Apprentissage et évolution des } \\
\text { besoins }\end{array}$ & $\begin{array}{l}\text { Marqueurs de qualification dans } \\
\text { un profil }\end{array}$ \\
\hline $\begin{array}{l}\text { Dynamique des } \\
\text { profils }\end{array}$ & $\begin{array}{l}\text { Apprentissage et évolution des } \\
\text { centres d'intérêt (cf. profils de } \\
\text { Bartle, 1996) }\end{array}$ & $\begin{array}{l}\text { Marqueurs de qualification dans } \\
\text { un profil }\end{array}$ \\
\hline $\begin{array}{l}\text { Réponses } \\
\text { cognitives } \\
\text { (Greenwald, 1968) }\end{array}$ & $\begin{array}{l}\text { Réactions à la communication } \\
\text { persuasive susceptibles de } \\
\text { traduire des changements } \\
\text { d'attitudes }\end{array}$ & $\begin{array}{l}\text { A voir : } \\
\text { Verbalisations } \\
\text { Indicateurs basés sur des } \\
\text { réponses physiologiques }\end{array}$ \\
\hline
\end{tabular}

\subsection{Défi 4 : élaborer une méthodologie de conception adaptée}

En tant qu' « architecte du changement ${ }^{29}$, le concepteur d'un SLP dispose dans sa boîte à outils d'un ensemble de principes de jeu et de persuasion qu'il peut implémenter. La démarche de conception devrait s'appuyer sur un processus structuré en phases successives autour d'objectifs bien définis avec des techniques, des outils spécifiques à chacune des phases. Malheureusement, les décisions de conception, la justification des options prises sont rarement documentées comme le montrent les quelques études de synthèse qui sont publiées. Par exemple :

\footnotetext{
${ }^{29}$ Terminologie dérivée du marketing : http://www.futurenowinc.com/persuasion_architecture.htm
} 
- Un état de l'art des systèmes persuasifs a été établi en 2009 par Torning et OinasKukkonen en utilisant leur modèle PSD comme grille d'analyse. La revue d'un corpus de 51 full-papers issus des conférences Persuasive Technology de 2006 à 2008 montre que certains principes sont utilisés de façon préférentielle ${ }^{30}$ sans que l'on sache pourquoi et que les considérations éthiques ne sont pas traitées.

- Une revue récente des travaux d'évaluation de la ludification établie par Hamari et al. (2014) montre qu'il n'est pas possible de conclure de façon définitive à propos de l'efficacité de ces techniques du fait de limites méthodologiques liées, entre autres, à l'utilisation de petits échantillons, à l'absence de groupe de contrôle, aux courtes périodes d'évaluation. De plus, bien que la gamification soit souvent présentée comme une avancée du marketing, très peu d'évaluations ont été effectuées dans ce domaine et la relation entre usage d'un système "gamifié " et comportement d'achat ne semble donc pas documentée.

La plupart des travaux concernant les systèmes persuasifs restent en fait assez évasifs à propos de la façon dont ils ont été élaborés, de la justification des principes utilisés et très peu d'informations sont données sur la démarche suivie pour la conception proprement dite (Nurulhuda et al. 2013). Deux aspects des pratiques actuelles sont successivement abordés ci-dessous :

- Le caractère empirique de la démarche de conception ;

- L'absence de mode d'emploi de la boîte à outils.

\subsubsection{Une démarche de conception actuellement plutôt empirique}

Les méthodologies de conception de logiciels interactifs ont suivi des évolutions continues depuis les premières méthodes d'analyse des systèmes d'information (Merise, SADT, UML, ...) et de nombreux modèles (linéaires, en $V$, en cycles, ...) ont été proposés avec un focus qui s'est progressivement déplacé sur l'utilisateur pour lui accorder un rôle devenu central. De plus, le caractère itératif et incrémental de la conception a conduit à l'émergence d'approches pragmatiques alternant les phases de développement et de validation sur des cycles courts et débouchant sur une diversité de méthodes agiles (RAD, XP, Scrum, ...).

Bien qu'il n'y ait aucune référence explicite à une méthode agile spécifique, les travaux conduits dans le domaine des jeux ou celui des systèmes persuasifs semblent plus proches de cette philosophie de développement que des approches classiques basées sur un cahier des charges détaillé. Par exemple, Fogg et Eckel (2007) ${ }^{31}$ recommandent d'appuyer la conception d'un système persuasif sur une analyse de dispositifs existant pour identifier des "patrons de persuasion " (persuasion pattern) efficaces puis de procéder par petits pas en suivant un modèle de développement basé sur un de ces patrons. Celui qu'ils ont identifié comporte 3 étapes traduisant des niveaux d'engagement de l'utilisateur d'un site : découverte, implication superficielle, véritable engagement. Les tâches successives de l'utilisateur associées à chaque étape (apprendre, visiter, essayer, démarrer, créer de la valeur, impliquer les autres, rester actif et loyal) constituent pour le concepteur des exigences à satisfaire qui orientent la stratégie de persuasion. Cette structuration de la conception en objectifs bien identifiés ne dit cependant rien de la nature des ressources de persuasion à mobiliser à chaque étape et ne prend pas en compte les variables de distance à la cible ou de sensibilité aux principes de persuasion évoqués ci-dessus (cf. section 3.2.1 et 3.2.2).

\footnotetext{
${ }^{30}$ Les 4 principes les plus utilisés sont le tailoring, la comparaison sociale, le tunneling et la réduction de la complexité.

31 Fogg B.J.and Eckles D. (2007) The Behavior Chain for Online Participation: How Successful Web Services Structure Persuasion, Y. de Kort et al. (Eds.): PERSUASIVE 2007, LNCS 4744, pp. 199-209, 2007. Springer-Verlag Berlin Heidelberg
} 


\subsubsection{Une boîte à outil sans véritable mode d'emploi}

Les différents principes ludo-persuasifs d'une boîte à outils sont plus ou moins adaptés en fonction de l'objectif de changement visé. De plus, les principes ne peuvent pas être tous implémentés dans un même dispositif : Kaptein (2012) a montré que l'utilisation de quelques principes est plus efficace qu'une implémentation systématique ${ }^{32}$. II est donc nécessaire de choisir parmi les principes ceux qui sont les plus adaptés, mais quelle correspondance établir entre tel principe et tel comportement cible? Quelles propriétés doivent être prises en compte ? Y a - t-il des conditions d'utilisation des principes en fonction des caractéristiques de la population cible, d'exigences particulières....? Quelles caractéristiques d'un contexte applicatif peuvent déterminer le choix des principes et/ou les ajustements qui sont requis pour améliorer l'efficacité de la persuasion?

La littérature de la persuasion technologique ne comporte pas de recommandation précise ni de guide de conception facilitant la sélection, l'articulation et l'implémentation des principes à mettre en œuvre pour atteindre les objectifs définis pour un projet particulier. Actuellement, de fait, la boîte à outils de conception est dépourvue de mode d'emploi. Tout au plus trouve-t-on dans la littérature un ensemble d'heuristiques, pour la plupart assez générales pouvant guider la conception :

- la « chaine comportementale de participation » mentionnée ci-dessus (Fogg \& Eckel, 2007) décrit un patron de persuasion qui peut être adapté à d'autres contextes ;

- le modèle FBM (Fogg, 2009) permet, éventuellement, de comprendre a posteriori pourquoi un comportement attendu n'a pas été mis en œuvre.

- le Behavior Wizard, (Fogg \& Hreha, 2010) aide à spécifier le type de changement recherché et fournit les pointeurs vers les ressources (théories et réalisations) adéquates ;

- Pour Oinas-Kukkonen et Hajumaa (2009) qui cherchent explicitement à structurer la démarche de conception, la route vers le changement comportemental passe par des heuristiques concernant: l'explicitation des enjeux sous-jacents au système persuasif, l'analyse du contexte de persuasion (type de changement, contexte d'usage, contexte utilisateur et stratégie définie par le message à faire passer), des spécifications du système organisées en étapes de développement (très générales et plutôt classiques) et enfin une organisation des principes de persuasion en 4 classes fonctionnelles, très structurantes (support à la tâche, au dialogue, à la crédibilité et à la socialité).

Sur la base des remarques précédentes, trois pistes prioritaires peuvent être envisagées pour faciliter la conception :

- L'établissement d'un catalogue de principes faisant consensus ;

- La validation de référentiel de qualité pour les composantes persuasives et ludiques ;

- L'adoption d'une approche contextuelle de la persuasion et de la ludification (contextual persuasive and gamification design).

\subsection{Défi 5 : développer des instruments d'analyse et de mesure appropriés}

Le pari des systèmes ludo-persuasifs est d'articuler les principes ludiques à ceux de la persuasion de telle sorte que l'utilisation du système interactif ne soit pas perçue comme une corvée mais qu'elle devienne agréable et que le rythme des interactions lors des usages du dispositif accompagne de façon cohérente la dynamique de changement de comportement. L'évaluation d'un SLP doit alors s'intéresser autant à sa composante ludique qu'à sa composante persuasive ainsi qu'à leurs interactions ce qui en fait un objet d'analyse singulier. Les questions d'évaluation des dispositifs interactifs ne sont pas nouvelles et

32 http://johnnyholland.org/2012/01/persuasion-profiling-attending-to-individual-differences-in-responses-to-persuasion-principles/ 
depuis les premiers efforts d'élaboration d'une véritable ingénierie ${ }^{33}$ de l'évaluation (Nielsen 1993, Kolski, 1993), la courte histoire de la discipline a été marquée par une succession de propositions de méthodes régulièrement revisitées pour faire face aux évolutions technologiques (voir Madan et al., 2012 pour un panorama récent). Dans quelle mesure les protocoles et les techniques de mesurage habituelles en IHM sont-ils bien adaptés aux systèmes ludo-persuasifs et quelles exigences spécifiques faut-il satisfaire pour répondre aux questions posées par l'évaluation de ces dispositifs ? Les sections ci-dessous donnent un aperçu rapide des travaux qui ont cherché à répondre à ces questions en abordant successivement trois dimensions d'évaluation des SLP : leur utilisabilité, leur " persuabilité » et leur « récréativité ».

\subsection{1 Évaluation de l'utilisabilité des SLP}

Les difficultés d'utilisation d'un SLP constituent un obstacle majeur à l'efficacité de la persuasion et au plaisir que l'on peut prendre à jouer. Elles peuvent être évitées en appliquant, dès la conception, les recommandations classiques d'ergonomie des dispositifs interactifs et l'identification des défauts qui persistent peut quant à elle s'appuyer sur les protocoles classiques d'évaluation d'IHM, (journaux, enquêtes, logs, analyse synchronique/diachronique,...). On trouvera dans http://www.gamasutra.com/view/feature/130745/better games through usability .php

l'illustration d'une évaluation experte et de test utilisateur dans le domaine des jeux vidéo. Cependant, les indicateurs et les critères de qualité usuels en ergonomie des IHM doivent être enrichis car utilité et facilité d'utilisation ne sont plus les dimensions principales de l'acceptabilité. II est en effet nécessaire d'adapter les grilles d'utilisabilité aux SLP car, les systèmes persuasifs, comme d'ailleurs les réseaux sociaux ou les jeux vidéo, n'ont pas pour finalité d'améliorer des performances de production et de faciliter la réalisation de tâches bien définies. Dès lors, certains indicateurs de qualité sont moins appropriés ; par exemple : le "temps mis à réaliser la tâche" perd son sens dès lors que l'objectif poursuivi est d'engager l'utilisateur dans le "flow" et de l'y maintenir.

\subsection{2 Évaluation de la dimension persuasive}

L'évaluation de la dimension persuasive d'un SLP pose des questions différentes selon le stade de son cycle de vie :

- Une évaluation avant son utilisation cherche à déterminer le potentiel persuasif du dispositif : quelle stratégie persuasive est mise en œuvre ? Quels principes de persuasion sont implémentés pour déterminer l'engagement et maintenir l'intérêt ?

- Après l'utilisation, il s'agit de déterminer quelle a été l'efficacité du dispositif et quels changements d'attitudes ou de comportements se sont produits.

Évaluation ex ante du potentiel persuasif d'un SLP. L'évaluation a priori de la "persuabilité » d'un dispositif interactif supposerait en premier lieu que l'on ait un référentiel adéquat permettant de lister sans ambiguïté les principes qui y sont mis en œuvre. Malheureusement, il n'y a pas pour l'instant de consensus à propos du contenu et de la structure de ce référentiel. Les différents modèles postérieurs aux propositions initiales de Fogg (Oinas-Kukkonen et Hajumaa, 2009, Némery, 2012, Cialdini, 2004...) présentent des listes de principes hétérogènes, parfois contradictoires et il est difficile d'en dégager une vue cohérente. Un aspect du défi est alors de stabiliser la liste des dimensions d'analyse et d'établir un catalogue normalisé de principes de persuasion. Sur cette base, il deviendra possible, comme c'est le cas en ergonomie des IHM, d'élaborer des outils structurés tels que des checkist, de quantifier et de valider des critères de qualité, de construire des échelles de

\footnotetext{
33 L'ingénierie de l'utilisabilité est une discipline située à l'intersection du génie logiciel et de l'interaction homme-machine. Elle emprunte ses outils, ses notions, concepts et méthodes à chacun de ces champs de recherche en cherchant à les articuler de sorte à optimiser les interactions hommes-machine en rendant les applicatifs plus faciles à utiliser.
} 
gravité de problème et finalement d'identifier des défauts, des faiblesses, des erreurs d'implémentation de principes de persuasion, voire de calculer des scores ${ }^{34}$.Les propositions faites dans Negri et Senach, (2015) vont dans ce sens.

Les méthodes d'inspection d'IHM (évaluation heuristique, inspection cognitive, ....) sont très populaires malgré leurs faiblesses méthodologiques et statistiques. Ces méthodes économiques d'évaluation a priori de la qualité ergonomique évitent le recours à des tests utilisateurs qui sont très coûteux (Nielsen et Molich, 1990). Elles s'appuient sur des heuristiques (cf. par exemple la grille d'analyse de Bastien et Scapin, 1997 en s'assurant que les exigences concernant par exemple la densité d'information, la cohérence ... sont satisfaites. Du côté des SLP, peu de travaux ont pour l'instant cherché à enrichir la boîte à outils avec des heuristiques susceptibles de guider la conception et l'évaluation. On peut citer Kientz et al. (2010) qui, dans le domaine de la santé, proposent 10 heuristiques de conception facilitant l'identification de défauts susceptibles de réduire l'efficacité de la persuasion. Les auteurs préconisent entre autres de recourir à des renforcements positifs lors de l'atteinte des objectifs et de bannir les renforcements négatifs, de laisser à l'utilisateur le contrôle des données privées, et d'assurer la transparence de la diffusion des données personnelles, ...). Ces recommandations restent très limitées et un travail considérable devra être réalisé avant que l'on puisse "persuader à moindre coût " comme on sait pratiquer l'ergonomie à bon marché (discount usability). Les travaux d'Andrew et al. (2007) sont une bonne illustration des efforts à entreprendre pour y parvenir : pour améliorer l'efficacité des suggestions dans les contextes de mobilité, ces auteurs proposent une grille d'analyse de l'opportunité, de la pertinence et de l'adéquation du contenu des suggestions.

Les heuristiques étant le produit d'une grande diversité d'expériences empiriques, nombre de travaux expérimentaux devront être mis en œuvre avant que l'on puisse identifier les principes génériques à satisfaire pour que les dispositifs interactifs deviennent vraiment persuasifs.

Évaluation ex post de l'efficacité persuasive des SLP : quels indicateurs de changement? La finalité des dispositifs interactifs étant de produire un changement d'attitude et/ou de comportement, il convient de définir les métriques les plus adaptées pour en mesurer l'efficacité et mettre en évidence les modifications au regard d'une situation antérieure. La réalisation d'enquête auprès des individus pour savoir s'ils ont adopté ou non le comportement prôné par une campagne de sensibilisation est le moyen le plus direct d'obtenir des réponses, mais les multiples biais des déclarations subjectives en font la méthode la moins fiable. L'identification d'indicateurs précis et la définition de métriques étant essentielles au développement scientifique d'une discipline, le paradigme d'évaluation basé sur la comparaison de données recueillies avant/après la mise en œuvre de la persuasion est privilégié.

Lorsque le changement comportemental visé peut être associé à des performances quantifiables, un recueil préalable est effectué auprès de la population cible et, les données sont comparées aux réponses produites après exposition plus ou moins longue aux messages persuasifs. En cas d'expérimentation sur plusieurs mois, un groupe contrôle permet généralement de s'assurer que les évolutions enregistrées ne sont pas liées à des facteurs externes. De bonnes illustrations de cette approche peuvent être trouvées dans l'étude classique de Mann et al $(2000)^{35}$ consacrée au tabagisme et dans Renaud et al. (2011) concernant l'évaluation de l'efficacité d'un jeu éducatif concernant l'asthme.

\footnotetext{
34 Un outil qui permettrait de pondérer les différentes dimensions d'évaluation serait très adapté. Cet outil pourrait être une simple feuille de tableur comportant la grille de critères d'analyse (voir par exemple la proposition de Maïs et Senach, 1994 pour le benchmarking de générateur d'interfaces homme-machine).

${ }^{35}$ Ce projet visant à réduire la consommation de tabac chez les jeunes, conduit sur une durée de 8 ans, n'a pas montré d'influence des techniques de persuasion utilisées.
} 
Les indicateurs utilisés pour apprécier l'impact de la persuasion sont le plus souvent très liés au domaine applicatif : réduction de facture pour la consommation énergétique, perte de poids pour l'adoption de nouveaux comportements alimentaires, calories consommées, distance parcourues, intensité des efforts... pour les incitations à l'exercice physique. Lorsque les performances peuvent être tracées et associées à l'utilisation du dispositif persuasif comme c'est notamment le cas dans les sites de commerce en ligne, les logs (par exemple nombre de visites, fréquence de consultation, visiteurs uniques, rebonds, ....) permettent d'appréhender directement son efficacité ${ }^{36}$. L'augmentation du taux de conversion traduit ainsi la capacité persuasive d'une campagne commerciale, la variation du nombre d'achats pouvant être directement associée à la mise en ligne d'une annonce commerciale. Certains auteurs (Guerini et al., 2010) vont même jusqu'à proposer de faire d'AdWords $^{37}$, la régie publicitaire de Google, une plateforme d'expérimentation permettant de tester à grande échelle l'efficacité de messages persuasifs.

Les traces d'usage ne sont pas toujours disponibles et ne sont pas toujours non plus les meilleurs indicateurs de changement. Des variables ad hoc, très spécifiques du contexte, sont alors souvent utilisées. Le défi à relever est ici d'identifier un paradigme transversal et une métrique générique indépendante du domaine applicatif. Une piste est proposée par Andrews et Manandhar (2009) qui, en s'appuyant sur la théorie de l'action raisonnée (Ajzen \& Fishbein, 1980) apprécient l'impact persuasif en s'intéressant aux changements d'opinion. Ils ont recours à des tâches d'ordonnancement dans lesquelles les sujets doivent indiquer l'importance relative d'un ensemble d'items. Le rang alloué à chaque item est interprété comme un indicateur de la valeur qui lui est accordée et les modifications enregistrées après exposition au dispositif persuasif traduisent l'impact qu'il a pu avoir.

\subsection{3 Évaluation de la dimension récréative}

Dans un dispositif ludo-persuasif, le caractère ludique de l'interaction est censé agir sur la motivation qu'a l'utilisateur à atteindre les objectifs qu'il poursuit. Le plaisir qu'il prend lors des interactions avec le dispositif le fait revenir dans un environnement dans lequel il est de nouveau exposé aux principes de persuasion, ce faisant, il y devient plus sensible et finit par agir dans la direction souhaitée. Les «incitations motivationnelles » (motivational affordances) implémentées sont constituées de récompenses (points, badges), d'une progression en niveaux, de feedback et de tableaux de bord permettant de suivre les performances... Mais cet arsenal de techniques est-il vraiment efficace, et la gamification conduit-elle à adopter le comportement cible ? Ces questions ont été abordées récemment dans une revue systématique des travaux d'évaluation de l'efficacité d'applications gamifiées (Hamari et al., 2014, cf. § 3.4). Les 24 études passées au crible ont été conduites dans une diversité de contextes applicatifs, et l'analyse examine le type d'incitateur utilisé, leurs impacts du point de vue psychologique (amélioration de la confiance, de l'estime de soi ...) et comportemental (variation des performances). II en ressort, que si des effets positifs sont mentionnés, ils sont largement dépendants du contexte applicatif et des caractéristiques des utilisateurs. De plus, outre les faiblesses méthodologiques classiques des études empiriques (taille d'échantillon insuffisante, absence de groupe contrôle, ...), la durée des expérimentations ne permet pas la plupart du temps de juger les effets à long terme.

L'évaluation de la dimension récréative pose des défis équivalents à ceux de la dimension persuasive : nécessité d'élaborer un référentiel, d'identifier des heuristiques et des bonnes pratiques et de définir des indicateurs pertinents. Sur ce dernier point, l'évaluation de la dimension hédonique est particulièrement importante. La qualité de l'expérience de

\footnotetext{
${ }^{36}$ Les logs sont aussi utilisés dans les applications gamifiées visant à renforcer la loyauté des visiteurs des sites de commerce en ligne (cf. http://badgeville.com/ )
}

37 http://fr.wikipedia.org/wiki/AdWords 
I'utilisateur devenant essentielle, il est nécessaire de pouvoir en analyser les composantes émotionnelles et sensorielles (Hassenzahl \& Tractinsky, 2006). Les efforts de formalisation de la notion d'expérience utilisateur (Bargas et al. 2011. Roto et al, 2011, Scapin et al., 2012; Pallot \& Pawar, 2012) et les définitions d'indicateurs opérationnels.

\section{PISTES DE TRAVAIL ET RESSOURCES POUR LA CONCEPTION DES SLP}

\section{1 Éléments pour une feuille de route}

Les défis et les questions qui viennent d'être abordées sont rappelés dans le schéma suivant.

Figure 8 : Rappel des défis et des pistes de travail

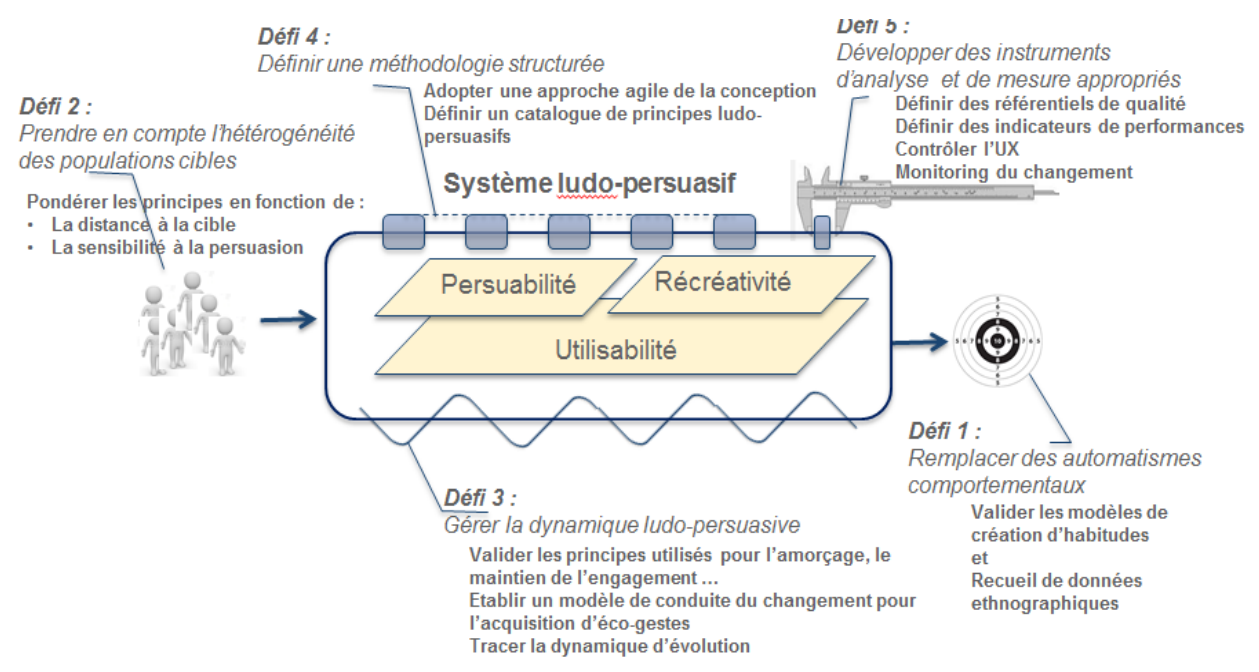

II ressort globalement de la discussion des défis que l'amélioration de l'efficacité des SLP passe par une validation des outils et une meilleure compréhension du contexte dans lequel est envisagé le changement comportemental :

- Validation des modèles d'acquisitions d'habitudes : modalités d'implémentation, leviers à utiliser, efficacité, ...

- Meilleure connaissance des bonnes et des mauvaises pratiques en matière de développement durable à partir de données ethnographiques : structuration des mauvaises habitudes, modalités et conditions de mise en œuvre, déclencheurs, bénéfices associés....

- Meilleure connaissance de la population cible, en l'occurrence être en mesure d'identifier la distance d'un individu ou d'une population par rapport au comportement à acquérir, et pouvoir déterminer quels leviers sont susceptibles d'être les plus efficaces, compte tenu de cette distance.

- Validation des principes utilisés pour la conduite du changement et des étapes de progression vers le comportement cible.

- Meilleure connaissance de la dynamique du changement : capacité à tracer l'évolution vers la cible à partir des interactions, à identifier des trajectoires individuelles, des patterns persuasifs.

- Validation d'une démarche de conception agile et "normalisation" d'une boite à outils de conception, notamment avec l'élaboration d'un catalogue consolidé de principes ludopersuasifs.

- Meilleure connaissance de ce qui fait la qualité et l'efficacité d'un système ludopersuasif: qualité intrinsèque estimée à partir de référentiels adéquats, mesure d'efficacité à partir d'indicateurs de performances, qualité de l'expérience utilisateur.... 
Des avancées sur ces éléments pourraient contribuer à l'établissement d'une ingénierie des systèmes ludo-persuasifs, mais à ces briques élémentaires, il faudrait associer une démarche structurée et celle-ci peut être recherchée par exemple en s'appuyant sur des approches existantes.

\subsection{Ressources méthodologiques pour la conception des SLP}

\subsubsection{Pour une approche contextuelle des SLP}

Un couplage opératoire des champs de recherche persuasion/ludification dans le contexte du développement durable devrait articuler une jouabilité (un gameplay) aux différents leviers d'incitation et de persuasion mobilisés au cours de la progression vers l'objectif. En pratique, ce couplage devrait déboucher sur des dispositifs permettant de prendre plaisir à l'adoption des comportements qui permettront les économies d'énergie. Les défis discutés ci-dessus (section 3.1 à 3.5 ) ont illustré la complexité et la diversité des questions à traiter ainsi que les fortes exigences à satisfaire pour que l'on puisse espérer une plus grande efficacité de ces dispositifs. II reste à déterminer comment on peut aborder leur conception. La démarche structurée de la conception contextuelle (contextual design) proposé par Beyer et Holtzblatt $\left(1997^{38}\right)$ a constitué une avancée importante pour l'ingénierie des IHM. Cette démarche peut être associée aux nouvelles approches de conception de services (service design, voir par exemple Schneider, 2012, Afsa, 2013) ${ }^{39}$ pour appuyer les premières phases de conception d'un système ludo-persuasif sur la mise en œuvre de techniques usuelles de conception centrée sur les utilisateurs. La représentation que I'on peut en donner est la suivante.

Figure 9 : Une démarche pour la conception contextualisée d'un SLP

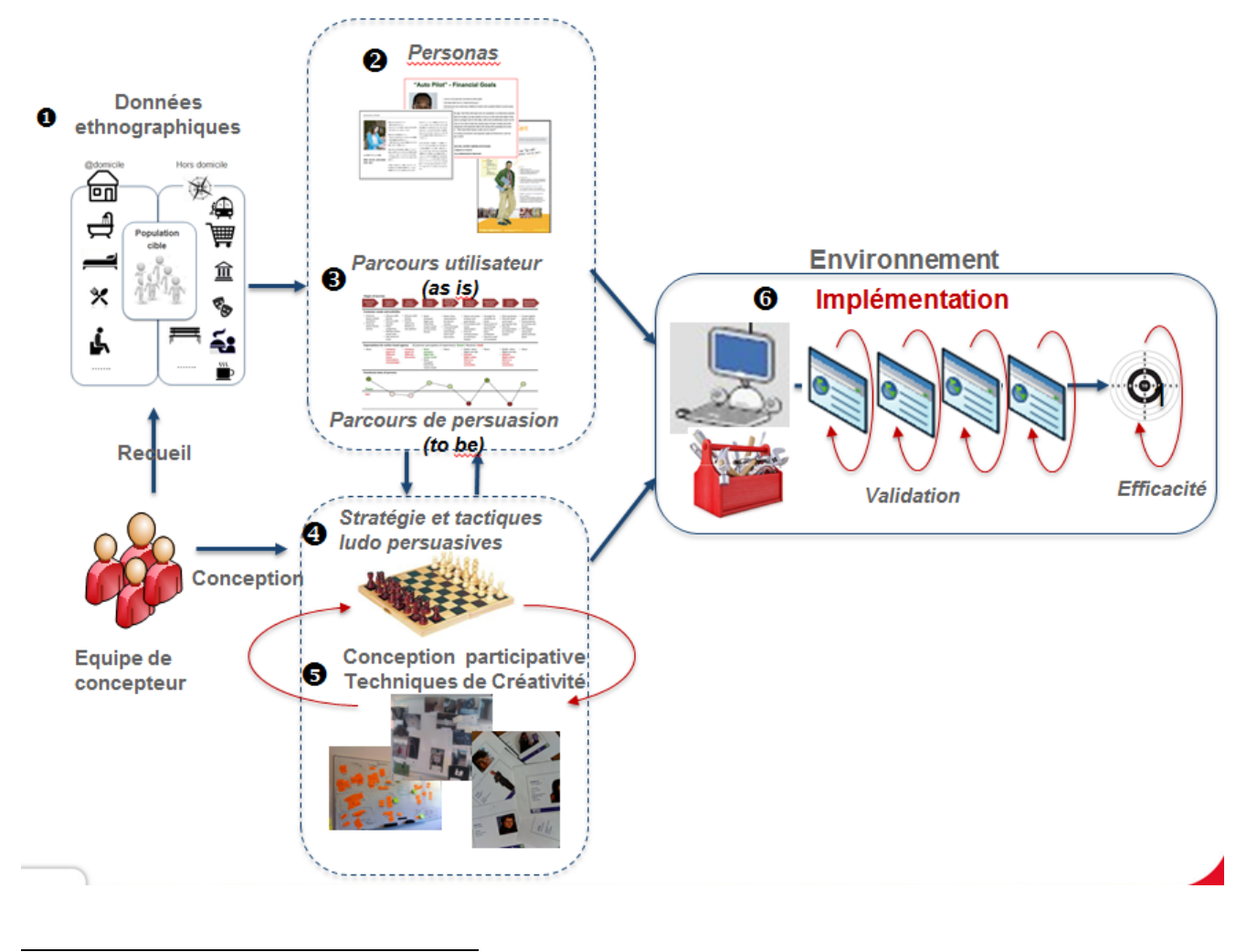

${ }^{38}$ voir aussi Holtzblatt, K., Wendell, J. B. \& Wood, S. (2005) Rapid Contextual Design http://gymnasium441.ru/files/ebook school/NO\%20SORT!!!/31/Morgan.Kaufmann.Rapid.Contextual.Design.A.How.to.Guide.to.Key.Techni ques.for.User.Centered.Design.pdf

${ }^{39}$ Voir aussi http://www.servicedesigntools.org/ 
En bref:

- A partir d'un projet initial spécifiant un cahier des charges minimal définissant l'objectif de changement comportemental et la population visée, la démarche peut s'appuyer sur un recueil de données ethnographiques (1) permettant d'identifier les différents contextes (domicile / extérieur) dans lesquels le comportement cible devrait être adopté et les pratiques réellement mises en œuvre par les individus. Le développement de l'internet des objets incite à envisager les systèmes ludo-persuasifs pour le développement durable comme étant à terme des jeux pervasifs (ARG Augmented Reality Game). La prise en compte de l'environnement naturel des activités pour concevoir le SLP constitue un premier pas dans cette direction.

Les données recueillies permettent d'élaborer:

- Des personas (2) caractérisant les sous populations identifiées ;

- Les "parcours clients" (3) (customer journey) constitués des différents points de contacts (touch point) associés au comportement à modifier ;

- L'élaboration de stratégies et de tactiques ludo-persuasives articulées aux parcours clients (4) et le recours (5) à des techniques de conception ouvertes (conception participative, créativité...) facilitent la conception de la composante ludique ;

- L'implémentation (6) est faite en adoptant une méthode agile et en mobilisant les techniques et outils de la boîte à outils. La validation est faite en intégrant le système ludo-persuasif dans l'environnement de l'utilisateur et en relation avec le parcours client. Par exemple, pour un système visant à faire adopter un équilibre alimentaire, les conseils, les recommandations, les aides diverses, devraient pouvoir prendre en compte l'environnement dans lequel évoluent les individus et leurs activités : contenu et utilisation du garde-manger, du frigidaire, points de contacts du parcours de l'affamé....

Les sections ci-dessous apportent des éclairages complémentaires sur les points suivants :

- Intérêt des personas pour les SLP ;

- Parcours de persuasion et design de service ;

- Recours aux méthodes participatives et créatives pour la ludification.

\subsubsection{Intérêt des personas pour les SLP}

Parmi les nombreux outils qui ont été développés pour l'utilisabilité des IHM, la modélisation des utilisateurs par des personas a constitué une avancée structurante (bien $q^{\prime}$ 'assez discutée ${ }^{40}$ ). On l'a dit, dans toutes les situations d'interaction, disposer d'une représentation de la cible constitue un avantage majeur pour améliorer l'impact des messages transmis et leur appropriation par les usagers. Les personas sont des modèles de classes d'utilisateurs (archétypes), élaborés à partir de recueil de données ethnographiques, qui permettent d'incarner la synthèse d'une collection de résultats d'études/questionnaires etc. (Cooper, 1998). Actuellement, la notion n'est pas explicitement mentionnée dans les travaux concernant la persuasion, mais on peut en trouver un équivalent indirect dans les critères d'expérience considérés par Fogg comme pertinents pour guider le choix des principes. En effet, le type d'objectif poursuivi (installer un nouveau comportement, réduire / augmenter la fréquence et/ou la durée du comportement cible...) et les canaux de

40 Voir par exemple Rönkko et al (2004) Personas is not applicable: Local Remedies Interpreted in a Wider Context , Proc. Participatory Design Conference, Toronto, Canada et plus récemment Bornet, $C$ et Brangier, E. (2013) La méthode des personas : principes, intérêts et limites, Bulletin de Psychologie, 2013/2, nº 524, 115-134 
communication associés sont des caractéristiques qui peuvent être définies dans un persona et utilisées pour construire la stratégie.

Des personas peuvent aider à capturer le contexte d'utilisation du dispositif et surtout les leviers à activer pour concrétiser le changement. Comme ils forment un petit groupe de persuadés "cible", il est également possible, dans ces cas précis, de construire un parcours de persuasion pour chaque persona afin d'identifier par la suite les points communs et les différences entre personas. Ces données peuvent ensuite être utilisées pour définir l'architecture de la persuasion et arbitrer notamment en fonction des priorités données aux différents personas (cible principale, secondaire etc).

En première analyse, outre les attitudes vis-à-vis des questions de développement durable, les attributs suivants seraient de bons candidats pour figurer dans des personas de SLP dédiés au développement durable :

- L'attitude face à la technologie, traditionnellement utilisée en ergonomie informatique pour la construction des personas est probablement importante également en ingénierie ludo-persuasive.

- Des dimensions plus intimes devraient figurer dans la construction des personas ludopersuasifs telles que : a) les valeurs de l'individu (voir par exemple Plex (Playful Experiences et l'impact des valeurs sur le ressenti de la récréativité, Korhonen et al., 2009 ) b) le sens (" meaning " comme souligné dans le modèle Octalysis) apporté à l'objectif de changement, c) l'intégration du persona dans un contexte social sont autant de guides qui permettent de poser des hypothèses sur les dynamiques et modalités de changement.

- la classification des types de joueurs de Bartle (1996) peut également être utilisée pour poser des hypothèses sur la dynamique de jeu et le choix des principes.

- D'une manière générale, les motivations et les expériences précédentes avec des dispositifs liés au changement de comportement contribueraient à enrichir ces personas.

\subsubsection{Design de service et parcours de persuasion}

La stratégie imposée par le concepteur d'un SLP pour guider l'adoption d'un comportement donné définit un parcours de persuasion qui doit être articulé à la logique d'activité des individus. Cette notion rejoint celle de " parcours client " utilisée dans le service design : il s'agit d'une cartographie structurée des interactions qu'un client peut avoir avec les différents dispositifs qui lui fournissent un service particulier. La succession des différents points de contact (touchpoint) à partir desquels les usagers interagissent avec le service (face à face, borne, site web, téléphone...) peut être décrite comme un parcours dans le service, une chronologie d'expériences dont les attributs émotionnels qualifient le ressenti de l'utilisateur lors des interactions. Cette approche, établie du point de vue du client, permet une compréhension macroscopique et globale des principaux facteurs influençant la qualité de l'expérience utilisateur et l'identification des points faibles sur lesquels il faut agir. La figure ci-dessous en donne une illustration pour un service de voyage en ligne. 
Figure 10 : Cartographie de parcours client dans un service de voyage en ligne ${ }^{41}$

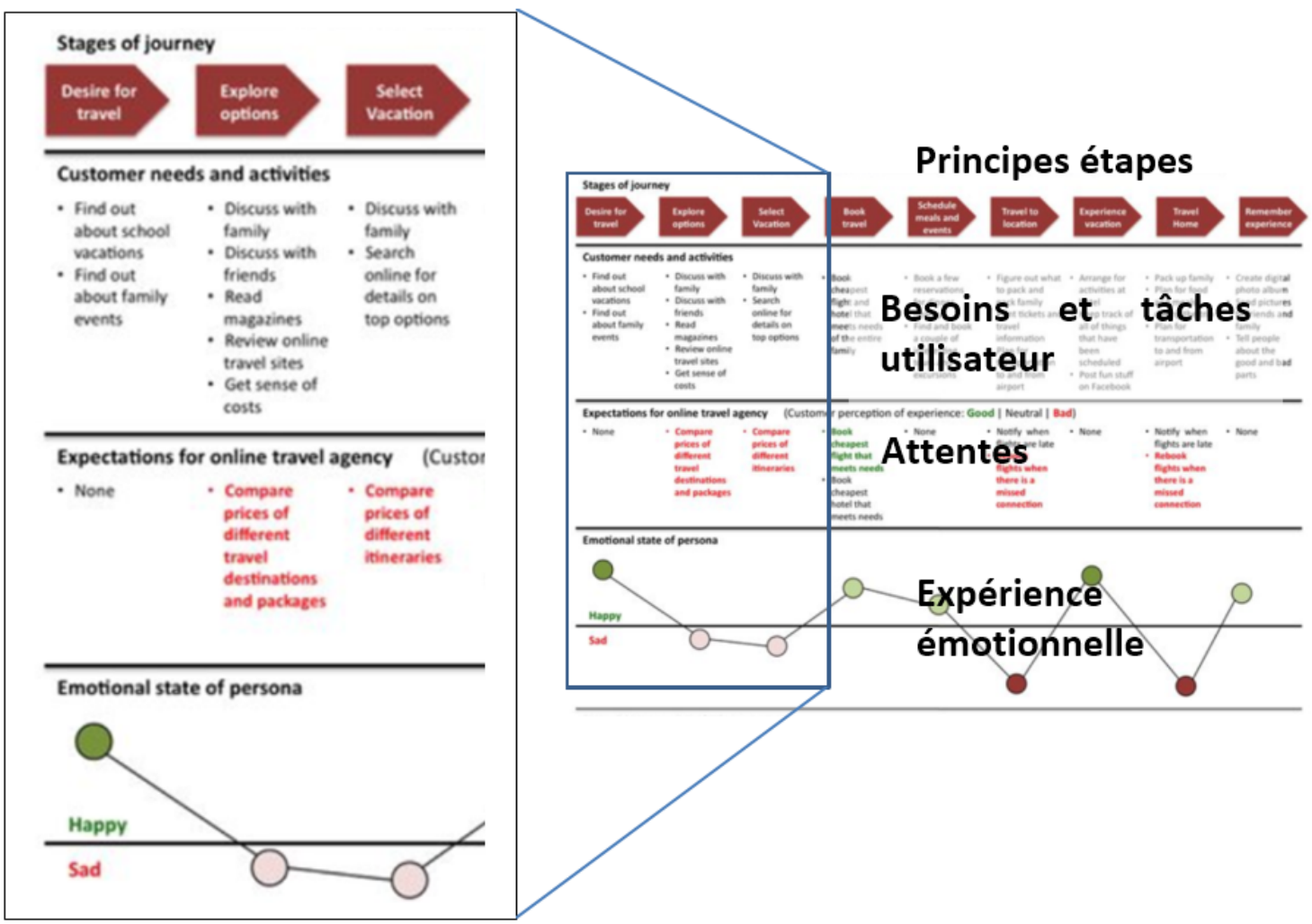

Source : $\quad$ http://experiencematters.wordpress.com/2013/04/03/seven-steps-fordeveloping-customer-journey-maps/

A l'instar de ces parcours clients qui identifient l'expérience utilisateur tout au long d'interactions multicanaux, on peut envisager de construire des parcours clients focalisés sur la persuasion et le changement de comportement. Cette approche permettrait :

- de poser des hypothèses sur les moments clés du processus de persuasion ;

- de clarifier les modalités d'utilisation des principes de persuasion ;

- d'identifier les indicateurs d'usage du dispositif aidant à l'évaluation de l'efficacité persuasive du dispositif.

\subsubsection{Méthodes participatives et créativité pour la ludification}

Les solutions pour le choix des principes sont parfois très ouvertes : par exemple, Zicherman et Cunningham (2011) citent Radoff qui considère que lancer des dés pour choisir les principes à mettre en œuvre lors de la création d'un jeu est une méthode acceptable. Ce recours au tirage au hasard laisse supposer que l'essentiel de la conception est ailleurs. On trouvera dans Djaouti et al (2010) une revue des méthodologie de Game Design qui recense les modèles formels (modèles du jeu, des joueurs, de la relation joueurjeu, du processus de conception) ainsi que les "recettes" associées et dans Lucero et Arrasvuori (2010) des évaluations d'un support matériel à la création de jeu (PLEX Cards). Outre les approches de conception spécifiques au domaine des jeux, dès lors que le développement des systèmes ludo-persuasifs est situé dans le cadre plus large de l'internet

\footnotetext{
${ }^{41}$ Le lecteur intéressé en trouvera de nombreuses illustrations dans : 
des objets, les techniques de conception participatives utilisées dans les projets d'innovation peuvent constituer des ressources appréciables. Par exemple, dans le projet Européen Elliot (Experiential Living Lab for Internet of Things) le couplage d'une méthode de bodystorming (Aloha !) avec une technique de génération d'idées basée sur l'utilisation de faux capteurs (Geniot) a permis d'explorer, sous des modalités très ludiques, des applications potentielles de l'internet des objets pour la qualité de l'air (Negri et al., 2012) ${ }^{42}$.

\section{CONCLUSION - APPROCHE HOLISTIQUE DU CHANGEMENT}

Dans quelle mesure la convergence des technologies persuasives avec celles du jeu peut-elle contribuer à faire évoluer plus rapidement les comportements de consommation ? Jusqu'à quel point, ces dispositifs "ludo-persuasifs" sont-ils susceptibles de faciliter des changements de comportements de leurs utilisateurs? Le tour d'horizon des travaux concernant les jeux et la persuasion présenté ci-dessus est bien sûr à la fois très rapide et dense. II a insisté sur le fait que si le rapprochement de ces deux technologies est encore à peine émergent, ce couplage est aussi très superficiel, ce qui compte pour beaucoup dans l'efficacité limitée des SLP actuels (Hamari et al., 2014). Le "buzz" existant autour de la ludification a été justifié par le potentiel du couplage des deux domaines, mais les déceptions restent fortes.

Parmi les démarches de conception qui paraissent les plus importantes, l'intégration d'un système ludo-persuasif dans son environnement d'utilisation, en cohérence avec la logique d'activité des utilisateurs est un aspect déterminant de son efficacité : un système développé "hors sol", indépendamment des modes de vie ne peut qu'avoir un impact très limité. Une des difficultés de changement dans le contexte du développement durable tient à ce qu'il s'agit de changer de mode vie plutôt que d'agir sur des comportements isolés et c'est bien à cette approche holistique que s'attelle le mouvement des villes en Transition ${ }^{43} \mathrm{Au}$ niveau individuel, les travaux convergent pour montrer qu'un comportement donné est le produit de variables internes de l'individu et d'influences provenant de l'environnement, comme l'illustre le schéma ci-dessous.

\footnotetext{
42 Voir aussi le projet Ecofamilies visant à travers une démarche de co-conception, une solution technologique innovante permettant d'encourager les comportements éco-responsables des familles (Decorme et al., 2012).

${ }^{43} \mathrm{http}: / /$ villesentransition.net/
} 


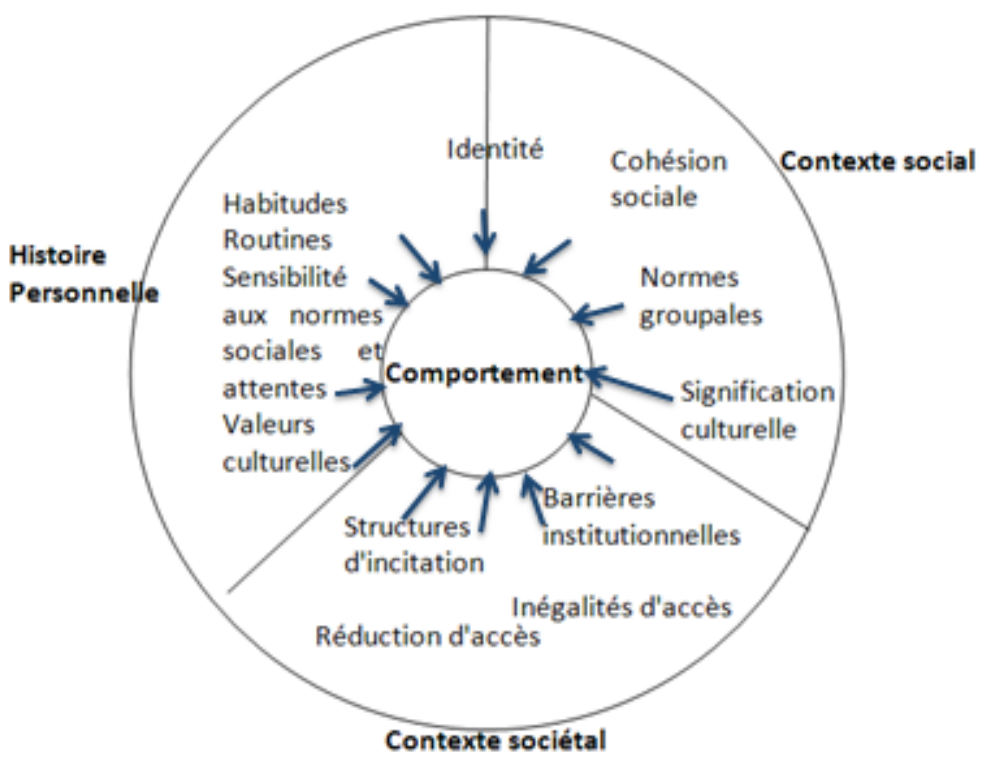

Ainsi, un comportement donné n'est jamais isolé : aux déterminants internes liés à I'histoire personnelle de l'individu (habitudes, routines, valeurs culturelles, croyances...) s'ajoutent des déterminants externes aussi bien physiques (proximité des poubelles de tri pour prendre un exemple trivial), sociaux (normes de groupes, signification culturelle ...) que sociétaux (structures d'incitation, barrières institutionnelles ...) qui modulent les décisions d'action.

Une idée forte qui a progressivement émergé des travaux conduits jusqu'à présent sur le changement en général et sur l'adoption d'éco-gestes en particulier est que finalement, l'implémentation effective d'un changement comportemental nécessiterait la mise en œuvre de leviers agissant aussi bien au niveau macroscopique sur les déterminants externes du comportement qu'au niveau microscopique. Malheureusement, ces deux approches sont souvent exclusives (Egmond et Bruel, 2007), et soit on privilégie les déterminants internes du comportement avec des solutions qui cherchent à agir sur les attitudes, la motivation, les habitudes et les normes personnelles, soit on s'intéresse plutôt aux déterminants externes : incitations fiscales, contraintes institutionnelles et pratiques sociales.

Les approches holistiques du changement comportemental combinant les deux points de vue n'étant pas la règle, ceci peut expliquer l'efficacité relativement faible des solutions généralement mises en œuvre jusqu'à présent. Lahlou (2008) propose une lecture complémentaire de cette situation dans laquelle le comportement individuel est piloté par un ensemble d'indices, de contraintes réparties entre 3 niveaux de détermination du comportement humain :

- Le niveau matériel : réalité physique et artéfacts - affordances et contraintes et incitation (par la fonction) ;

- Le niveau psychologique : implémentation d'habitudes et de représentations prédéfinies qui sont des interprétations possibles des situations (interprétation symbolique mais aussi comme en musique : interprétation d'exécution - L'acteur individuel et social est un interprète qui parfois joue faux) ;

- Le niveau institutionnel : régulation et maintenance de l'installation et de son usage.définition des règles d'usages, conventions, etc.

La confrontation à une situation donnée nécessite l'élaboration d'une représentation adaptée qui s'appuie sur des indices fournis par l'installation matérielle du monde, sur les scripts comportementaux disponibles en mémoire et sur les éventuels recadrages des institutions pour canaliser les pratiques et les réorienter. 
En attendant la cohérence de cette "installation du monde", il reste toujours possible de s'atteler à des tâches plus modestes et c'est ce que nous avons entrepris, d'abord en proposant, à la suite du travail qui vient d'être présenté une première élaboration d'une grille de principes ludo-persuasifs (cf Negri et Senach, 2015) et en la mettant ensuite à l'épreuve en réalisant une déconstruction d'un challenge écologique en entreprise (Senach et Negri, 2015).

\section{REFERENCES}

- Afsa, C. (2013). Design de Service. Cité du Design (Ed.)

- Ajsen, I., \& Fishbein, M. (1980). Understanding attitudes and predicting social behavior. Englewood Cliffs, NJ: Prentice-Hall.

- Kim, Amy Jo, (2000). Community Building on the Web: Secret Strategies for Successful Online Communities, Peachpit Press.

- Andrew, A., Borriello, G., \& Fogarty (2007). Toward a systematic understanding of suggestion tactics in persuasive technologies; PERSUASIVE'07 Proceedings of the 2nd international conference on Persuasive technology (pp. 259-270).

- Andrews, P., \& Manandhar, S. (2009). Measure of Belief Change as an Evaluation of Persuasion, in: Persuasive Technology and Digital Behaviour Intervention Symposium, Edinburgh, Scotland, April 6-9.

- Atkinson, B. M. C. (2006). Captology: A Critical Review, In W. I Jsselsteijn, \& al. (Eds.): PERSUASIVE 2006, LNCS 3962 (pp. 171- 182).

- Asakawa, K. (2004). Flow experience and autotelic personality in Japanese college students: How do they experience challenges in daily life? Journal of Happiness Studies, 5, (pp. 123-154).

- Bandler, R., \& Grinder, J. (1982). Les secrets de la communication, Reuille, Versoix. Suisse.

- Bach, C., \& Scapin, D.L. (2010). Comparing Inspections and User Testing for the Evaluation of Virtual Environments, Intl. Journal of human-computer interaction, 26(8), (2010, pp.786-824), Taylor \& Francis.

- Bandura, A. (1969). Principles of behavior modification. New York: Holt, Rinehart \& Winston

- Bang, M., Gustafsson, A,. \& Katzeff, C. (2007). Promoting New Patterns in Household Energy Consumption with Pervasive Learning Games. Proceedings PERSUASIVE'07, 2nd International Conference on Persuasive Technology, (pp. 55-63) Springer-Verlag Berlin, Heidelberg

- Bang, M., Torstensson, C., \& Katzeff, C. (2006). The PowerHouse: A persuasive computer game designed to raise awareness of domestic energy consumption. Proceedings PERSUASIVE'06, Proceedings of the 1st International Conference on Persuasive Technology, (pp. 123-132), Springer-Verlag Berlin, Heidelberg

- Bartle, R.A. (1996). Hearts, Clubs, Diamonds, Spades: Players Who Suit MUDs. Disponible en ligne le 22/06/2015: http://mud.co.uk/richard/hcds.htm

- Bartle, R.A. (2005). Virtual Worlds: Why People Play, In Massively Multiplayer Game Development 2, Thor Alexander (ed.), Charles River Media, Hingham MA. Disponible en ligne le 22/06/2015 : mud.co.uk/richard/VWWPP.pdf

- Bastien, C. (2012). Réchauffement climatique : les contributions possibles de la psychologie ergonomique et de l'interaction humain-machine à la réduction de la consommation d'énergie, Le travail humain, 3, vol. 75, P.U.F, (pp. 329 -348).

- Bastien, C., \& Calvary, G. (2013). PISTIL : Persuasive Interaction for SusTainabiLity.25ième conférence francophone sur l'Interaction Homme-Machine, IHM'13, Nov 2013, Bordeaux, France. http://hal.inria.fr/docs/00/88/09/31/PDF/4-2.pdf

- Scapin, D. L., \& Bastien, J. M. C. (1997). Ergonomic criteria for evaluating the ergonomic quality of interactive systems. Behaviour \& Information Technology, 16, 220-231. Berdichevsky, D., \& Neuenschwaner, E. (1999). Toward an Ethics of Persuasive Technology. Communications of the ACM, 42(5), 51-58

- Scapin, D., Senach, B., Trousse,B., Pallot, M. (2012). User Experience: Buzzword or New Paradigm?. Proceedings ACHI 2012, The Fifth International Conference on Advances in Computer-Human Interactions, Valencia, Spain, January 2012.

- Beyer, H., \& Holtzblatt, K. (1997). Contextual design: Defining Customer Centered Systems. Morgan Kaufman. 
- Boutaud, A. (2009). Ecologie - De la sensibilisation aux changements de comportement. Millénaire, Centre Ressources Prospective du Grand Lyon. Disponible en ligne le 22/06/15: http://www.millenaire3.com/fileadmin/user upload/syntheses/ecologie comportement.pdf

- Brög, W., Erl, E., \& Mense, N. (2002). Individualised Marketing Changing Travel Behaviour for a better Environment, Paper presented at the OECD Workshop: Environmentally Sustainable Transport. Berlin, Disponible en ligne le 22/06/15 : http://www.kontiv.de/info/IndiMark.pdf

- Caillois, R. (1992). Les jeux et les hommes : le masque et le vertige. Folio, Paris

- Cialdini, R.B., (2004). Influence et manipulation : Comprendre et maîtriser les mécanismes et les techniques de persuasion

- Coester, P., Vessilern, B., \& Collombat, H. (2002). Management de la mobilité par le "Marketing individualisé" - Une approche innovante pour changer durablement les comportements dans les déplacements quotidiens. Certu

- Cooper, A. (1998). The Inmates Are Running the Asylum - Why High Tech Products Drive Us Crazy and How to Restore the Sanity, Sams (ed.)

- Csikszentmihalyi, M. (1990). Flow, the Psychology of Optimal Experience, New York, Harper and Row.

- Csillik, A.S. (2009). Polémique actuelle autour du modèle transthéorique : ce modèle mérite-t-il d'être encore utilisé ? Annales Médico-Psychologiques, 167, (pp. 355-360), Elsevier Masson, France.

- Damasio, A.R. (1995). L'erreur de Descartes : la raison des émotions. Odile Jacob (ed.), Paris.

- Decorme, R., Thibault, E., \& Zarli, A. (2012). Energy Awareness In The Office And Residential Built Environments: The Ecoffices And Ecofamilies Experiments. In Proceedings of the CIB W78 2012: 29Th International Conference -Beirut, Lebanon, 17-19 October. Disponible en ligne le 22/06/15 : http://itc.scix.net/data/works/att/w78-2012-Paper-6.pdf

- Desurvire, H., Kondziela, J., \& Arwood, M. (1992). What is Gained and Lost When using Evaluation Methods Other Than Empirical Testing. In Proc. of CHI 1992, Cambridge University Press.

- Djaouti, D., Alvarez, J., \& Jessel, J. P. Concevoir l'interactivité ludique : une vue d'ensemble des méthodologies de " Game Design , IRIT, $n^{\circ} 27$, Disponible en ligne le 22/06/15: http://www.ludoscience.com/files/ressources/Methodologie Game Design.pdf

- Egmond, C., \& Bruel, R. (2007). Nothing is as practical as a good theory, analysis of theories and a tool for developing interventions to influence energy-related behaviour, SenterNovem, September 2007

- Eyal, N. \& Hoover, R. (2014). Hooked: How to Build Habit-Forming Products. Kindle Ebook.

- Fenouillet, F. (2012). Les théories de la motivation, Dunod (ed.), Paris.

- Fernandez, A., Insfran, E. \& Abrahao, S. (2011). Usability Evaluation Method for the Web: A Systematic Mapping Study, Information and Software Technology, 53, (pp. 789-817), Elsevier.

- Festinger, L., Riecken, H.W., \& Schachter, S. (1993). L'échec d'une prophétie, PUF,

- Fogg, B.J. (1998). Persuasive Computers: Perspectives and Research Directions. Proc. CHI 98, 18-23 April, Los Angeles, California, USA., (pp. 225-232).

- Fogg, B.J., \& Eckles, D. (2007). The Behavior Chain for Online Participation: How Successful Web Services Structure Persuasion. In Y. de Kort, \& al. (Eds): Persuasive 2007, LNCS 4744, (pp. 199-209), SpringerVerlag, Berlin Heidelberg.

- Fogg, B.J. (2009). A Behavior Model for Persuasive Design, Persuasive'09, April 26-29, Claremont, California, USA, ACM

- Fogg, B.J., \& Hreha, J. (2010). Behavior Wizard: A Method for Matching Target Behavior with Solutions, in T. Ploug, P. Hasle, H. Oinas-Kukkonen (Eds.): PERSUASIVE 2010, LNCS 6137, (pp. 117-131), 2010., Springer-Verlag Berlin Heidelberg

- Goldberg, L.R. (1990). An alternative "description of personality": The big-five factor structure. Journal of Personality and Social Psychology, 59, (pp. 1216-1229).

- Goldberg, L.R. (1993). The structure of phenotypic personality traits. American Psychologist, 48, (pp. 26-34).

- Goldstein, N.J. \& Cialdini, R.B. (2004). Social Influence: compliance and Conformity Annu. Rev. Psychol. 2004. 55, (pp. 591-621).

- Goldstein, N.J., Cialdini, R.B., \& Griskevicius, V. (2008). A room with a viewpoint: Using social norms to motivate environmental conservation in hotels. Journal of Consumer Research, 35, (pp. 472-482).

- Gray, W.D., \& Salzman, M.C. (1998). Damaged merchandise? A review of Experiments That Compare Usability Evaluation Methods. Human-Computer Interaction, 1998, vol.13, (pp. 203-26). 
- Greenwald, G.A. (1968). On Defining Attitude and Attitude Theory, in Anthony G. Greenwald, Timothy C. Brock, and Thomas M. Ostrom, (Eds.), Psychological Foundation of Attitude, New York: Academic Press.

- Guerini, M., Strappavara, C., \& Stock, O. (2010). Evaluation Metrics for Persuasive NLP with Google AdWords. in Proceedings of the Seventh International Conference on Language Resources and Evaluation (LREC'10), Valetta, Malta.

- Harjumaa, M., Segerstal, K,, \& Oinas-Kukkonen, H. (2009). Understanding persuasive software functionality in Practice: A field Trial of Polar FT60, Persuasive'09, April 26-29, Claremont, California, USA

- Hamari, J., kOIVISTO, J., \& Sarsa, H. (2014). Does Gamification Work? - A Litterature Review of Empirical Studies on Gamification. In Proceedings of the $47^{\text {th }}$ Hawaii International Conference on System Sciences, Hawaii, USA, January 6-9, 2014.

- Hovland, C., Janis, I.L., \& Handkelley. H. (1953). Communication and Persuasion: Psychological Studies of Opinion Change. New Haven: Yale UP,

- Huizinga, J. (1988). Homo Ludens - Essai sur la fonction sociale du jeu. Une étude de l'influence du jeu sur la culture européenne, Gallimard (Ed.), Paris

- Hunicke, R., Leblanc, M., \& Zubek (2004). MDA : a formal approach to Game Design and Game Research. In AAAl Workshop on Challenges in Game Artificial Intelligence. Disponible en ligne le 23/06/15: http://www.cs.northwestern.edu/ hunicke/MDA.pdf

- Jackson, T. (2005). Motivating Sustainable Consumption - a review of evidence on consumer behaviour and behavioural change. Centre for environmental Strategy, University of Surrey.

- Jeffries, R., \& Desurvire, H. (1992). Usability testing vs. heuristic evaluation: was there a contest? SIGCHI Bulletin, 24 (4), (pp. 39-41).

- Joule, R.V., \& Beauvois, J.L. (1999). La soumission librement consentie : Comment amener les gens à faire librement ce qu'ils doivent faire. Presses Universitaires de France.

- Kaptein, M. C., (2012). Personalized Persuasion in Ambient Intelligence. PhD, Eindhoven University of Technology. Disponible en ligne le 23/06/15: http://www.mauritskaptein.com/wpcontent/uploads/2010/12/PersonalizedPersuasion MauritsKaptein.pdf

- Kaptein , M.C., Markopoulos, P, De Ruyter, B., Aarts, E. (2010). Persuasion in ambient intelligence. J Ambient Intell Human Computer, 1, (pp. 43-56).

- Kim, H., \& Fesenmaier, D. R. (2008). Persuasive Design of Destinaton Web Sites: An Analysis of First Impression. In J. of Travel Research OnlineFirst.

- Kolski, C. (1993). Ingéniérie des interfaces hommes-machines, Conception et évaluation. Hermes (ed.), Paris.

- Korhonen H., Montola M., \& Arrasvuori J. (2009).Understanding Playful Experiences Through Digital Games.In Proc. of DPPI '09. (pp. 274-285).

- Kumar, Janaki Mythily, \& Herger, Mario (2013).: Gamification at Work: Designing Engaging Business Software. Aarhus, Denmark, The Interaction Design Foundation. ISBN: 978-87-92964-06-9.

- Lally, P. Van Jaarsveld, . H. H., Potts, H.W.W. \& Wardle, J. (2010). How are habits formed: Modeling habit formation in the real world. European Journal of Social Psychology. 40, 6 (pp. 998-1009), October 2010.

- Lewin, K. (1947). Group decision and social change. In T. Newcomb, E. Hartley (Eds.), Readings in Social Psychology. New York : Holt.

- Lockton, D., Harisson, D., \& Staton, N., (2010). The design with intent method: a design tool for influencing user behaviour. Applied Ergonomics, Vol.41 (No.3). (pp. 382-392).

- Lockton, D., Bowden, F., Green, C. Brass, C., \& Gheerawo, R. (2013). People and energy: A design-led approach to understanding everyday energy use behaviour. EPIC 2013 Proceedings, Vol 2013, Issue1, (pp. 348-362), September.

- Lucero, A., \& Arrasvuori, J. (2010). PLEX Cards: A Source of Inspiration When Designing for Playfulness. Proc. Fun and games '10, Proceedings of the $3^{\text {rd }}$ International Conference on Fun and Games.

- Levieux, G. (2011). Mesure de la difficulté des jeux video, CNAM, Paris. Disponible en ligne le 22/06/15: http://guillaumelevieux.com/siteperso/contents/thesisdefense/thesisDefense.php

- Lowry, P.B., Gaskin, J., Twyman, N., Hammer, B., \& Roberts T.L. (2013). Proposing the hedonic-motivation system adoption model (HMSAM) to increase understanding of adoption of hedonically motivated systems, Journal of the Association for Information Systems, vol. 14(11), (pp. 617-671).

- Madan, A., \& Dubey, S.K. (2012). Usability evaluation methods: a literature review. Int.J. of Engineering Science and technology (IJEST), Vol.4, $\mathrm{n}^{\circ} 2$, February 2012 
- Maïs, C., \& Senach, B. (1994). Evaluation comparative de Logiciels: une méthodologie de choix d'un GIHM, ERGO Al 94: Ergonomie et informatique avancée, (pp. 458-469).

- Mann S.L., Peterson, A.V., Jr, Marek, P. M., \& Kealey, K. A. 2000. The hutchinson smoking prevention project trial: design and baseline characteristics, Prevmed, Jun,; 30, 6, (pp. 485-95).

- Moreno, J.L. (1935). Les Fondements de la sociométrie, PUF, paris.

- Negri, A.-L, Trousse, B., \& Senach, B. (2012). Ideation of IoT Services with Citizen: Coupling GenloT and AloHa! Methods, in: ServDes'2012 - Service Design and Innovation Conference, Helsinki, Finlande. Disponible en ligne le 22/06/15 : http://www.ep.liu.se/ecp article/index.en.aspx?issue=067; article=017

- Negri, A.-L., \& Senach, B. (2015). Systèmes ludo-persuasifs pour la consommation durable - 2 - Proposition d'une grille de principes ludo-persuasifs. Journal d'Interaction Personne-Système - Vol. 4, Num. 1, Art. 7, Juin 2015. AFIHM.

- Nielsen, J. (1993). Usability Engineering. Morgan Kaufmann

- Nielsen, J., \& Molich, R. (1990). Heuristic Evaluation of User Interfaces, In Carrasco, J. \& Whiteside, J. (Eds.), Proceedings of ACM CHI'90 Conference on Human Factors in Computing Systems, ACM Press, (pp. 249-256).

- Nemery, A., Brangier, E., \& Kopp, S. (2011). First Validation of Persuasive Criteria for Designing and Evaluating the Social Influence of User Interfaces: Justification of a Guideline. In A. Marcus (Ed.): Design, User Experience, and Usability, Pt II, HCII 2011, LNCS 6770, (2011, pp. 616-624).

- Nemery, A. (2012). Elaboration, validation et application de la grille de critères de persuasion interactive. Thèse de $3^{\text {ème }}$ cycle, Université Paul Verlaine, Metz.

- Nemery, A., \& Brangier, E. (2014). Set of guidelines for persuasive Interfaces : organization and Validation of the Criteria. Journal of Usability Studies, Vol. 9, Issue 3, May 2014. Disponible en ligne le 22/06/15: http://uxpajournal.org/wp-content/uploads/pdf/JUS Nemery May 2014.pdf

- Nurulhuda, I., Shiratuddin, M.F., Wong, K. (2013). Persuasion Techniques for Tourism Website Design, International Conference on E-Technologies and Business on the Web (EBW2013), Bangkok, Thailand. (pp. 175-180).

- Oinas-Kukkonen, H., \& Harjumaa, M. (2008a). A Systematic Framework for Designing and Evaluating Persuasive Systems. In H. Oinas-Kukkonen et al. (Eds.); persuasive 2008, LNCS 5033, (pp. 164-176), Springer-Verlag.

- Oinas-Kukkonen, H., \& Harjumaa, M. (2008b). Towards Deeper Understanding of Persuasion in Software and Information Systems. First International Conference on Advances in ComputerHuman Interaction, (pp. 200-205).

- Oinas-Kukkonen, H., \& Harjumaa, M. (2009(. Persuasive Systems Design: Key Issues, Process Model, and System Features. The Communications of the Association for Information Systems, Vol. 24, 28,

- Macinnis, D.J., \& Jaworski, B.J. (1989). Information Processing from Advertisements: Toward an Integrative Framework. Journal of Marketing, 53, 4, (pp. 1-23)

- Prochaska, J.O., \& Diclemente, C.C. (2005). The Transtheorical Approach. In J. C. Norcross and M. R. Goldfried (eds.) Handbook of Psychotherapy Integration, Oxford University Press

- Radoff, J. (2011). Game On: Energize your Business with Social Games. Wiley.

- Vygotsky, L.S. (1978). Mind and society: The development of higher mental processes. Cambridge, MA: Harvard University Press.

- Renaud, L., Sauvé, L, \& Kaufman, D. (2011). Asthme : 1. 2 . 3... Respirez! Efficacité du jeu éducatif sur les attitudes à l'égard de l'asthme. Revue internationale Communication sociale et publique. Disponible en ligne le 22/06/15 : http://www.revuecsp.uqam.ca/numero/n6/pdf/RICSP RenaudEtAl 2011.pdf

- Rothschild, M.L. (1999). Carrots, Sticks, and Promises: A Conceptual Framework for the Management of Public Health and Social Issue Behaviors. Journal of Marketing, 63, 4, (pp. 24-37)

- Roto, V., Law, E., Vermeeren, A., \& Hoonhout, J. 2011. User Experience White Paper: Bringing clarity to the Concept of User Experience (Result from Dagstuhl Seminar on Demarcating User Experience, Sept., 15- 18, 2010). Disponible en ligne le 22/06/15 http://www.allaboutux.org/files/UX-WhitePaper.pdf

- Saparova, D. (2012). Motivating, Influencing, and persuading Patients through Personal health Records: A Scoping Review. Perspective in Health Information Management, Summer 2012.

- Schwartz, S. (2006). Les valeurs de base de la personne : théorie, mesures et applications. Revue française de sociologie, 2006/4 (Vol. 47), (pp. 929-968). Disponible en ligne le 22/06/15 : http://www.cairn.info/revuefrancaise-de-sociologie-2006-4-page-929.htm 
- Schneider, J. (2012). This is Service Design Thinking: Basics - Tools - Cases. BIS Publishers B.V. (Ed.).

- Senach, B., \& Negri, A.-L. (2015). Systèmes ludo-persuasifs pour la consommation durable - 3 - Analyse du potentiel ludo-persuasif d'un challenge énergétique en entreprise, Journal d'Interaction Personne-Système Vol. 4, Num. 1, Art. 8, Juin 2015. AFIHM.

- Thaler, R. H., \& Sunstein, C. R. (2008) Nudge : Improving Decisions about Health, Wealth, and Happiness, Yale University Press.

- Torning, K. Hall, C., \& Oinas-Kukkonen, H. (2009). Persuasive System design: State of the Art and Future Directions. In: proceedings of PERSUASIVE 2009, New York, USA, Vol 350.

- Watzlawick, P. (1967). Une logique de la communication, 1967, Point Seuil

- West, R. (2005(. Time for a change: putting the Transtheoretical (Stages of Change) Model to rest, in Addiction, $\quad \mathrm{n}^{\circ} 100, \quad$ (pp. 1036-1039). Disponible en ligne le 22/06/15: http://onlinelibrary.wiley.com/doi/10.1111/j.1360-0443.2005.01139.x/pdf

- Zichermann, G., \& Cunningham, C. (2011). Gamification by Design. O'Reilly Media (Ed.)

\section{BIOGRAPHIE}

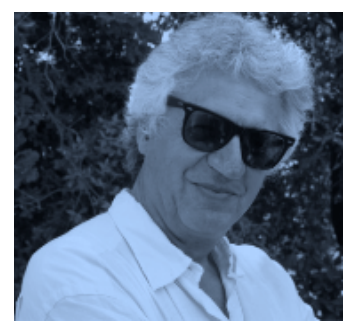

\section{Bernard SENACH}

a commencé ses activités en ergonomie cognitive chez INRIA en 1978 et jusqu'en 1990 s'est intéressé à la conception et à l'évaluation d'interfaces hommes-machines pour la conduite de processus dynamiques (centrales nucléaires, systèmes de transport ferroviaire). II crée en 1990 une des premières start-up d'INRIA qu'il codirige jusqu'en 2005. De retour dans la recherche, il travaille actuellement au CRISAM1 dans l'équipe Héphaïstos sur des projets de robotique d'assistance pour le maintien à domicile.

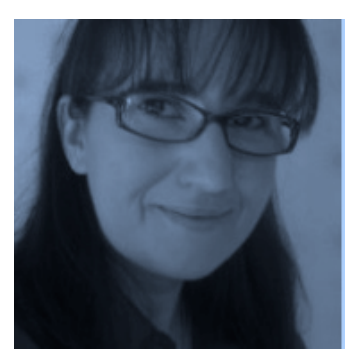

\section{Anne-Laure NEGRI}

est consultante indépendante et docteur en « Automatique Humaine » (Univ. de Valenciennes, 1999). Dans les années 90 , ses travaux de modélisation et de simulation des activités cognitives d'opérateurs des transports aériens l'orientent vers l'élaboration de dispositifs facilitant la prise de conscience des risques et l'adoption de comportements sécuritaires (pilotes de ligne, contrôleurs de vol). Depuis les années 2000, elle alterne les collaborations avec grands comptes, starts up ou structures académiques (Telecom Paristech, INRIA) confrontées aux problématiques d'utilisabilité, pertinence et viabilité d'innovations technologiques en les faisant bénéficier de ses compétences en ethnographie, ergonomie, co-création et sociologie des usages, notamment au travers de l'approche Design Thinking qu'elle co-enseigne depuis 2013 à the Sustainable Design School à Nice. 